\title{
Acknowledgement to Reviewers of IJERPH in 2017
}

\section{IJERPH Editorial Office}

MDPI AG, St. Alban-Anlage 66, 4052 Basel, Switzerland

Published: 11 January 2018

Peer review is an essential part in the publication process, ensuring that IJERPH maintains high quality standards for its published papers. In 2017, a total of 1594 papers were published in the journal. Thanks to the cooperation of our reviewers, the median time to first decision was 22 days and the median time to publication was 53 days. The editors would like to express their sincere gratitude to the following reviewers for their time and dedication in 2017:

Aadland, Eivind

Aasvang, Gunn Marit

Abballe, Annalisa

Abbott, Louise

AbiGhannam, Niveen

Abimanyi-Ochom, Julie

Ablewhite, Joanne

Aboud, Frances

Abraham, John P.

Abraham, K. J.

Abrams, David

Abrams, Stephen

Adam, Vojtech

Ádám, Balázs

Adamus-Białek, Wioletta

Addison, Clifton

Addo, O. Yaw

Adetona, Olorunfemi

Adini, Bruria

Adivar, Burcu

Adler-Nissen, Jens

Adoloratto, Giovanni

Afshari, Reza

Aggarwal, Anju

Aggazzotti, Gabriella

Agho, Kingsley

Agnisola, Claudio

Agodi, Antonella

Agrawal, Priyanka

Aguilar, Carme

Aguinaga-Ontoso, Inés

Ahern, Tracey

Ahmad, Hafiz Anwar

Ahmad, Azeem

Ahmed, Faruque

Ahmed, Zafar

Ai, Zhengtao

Aida, Jun

Aide, Michael T.

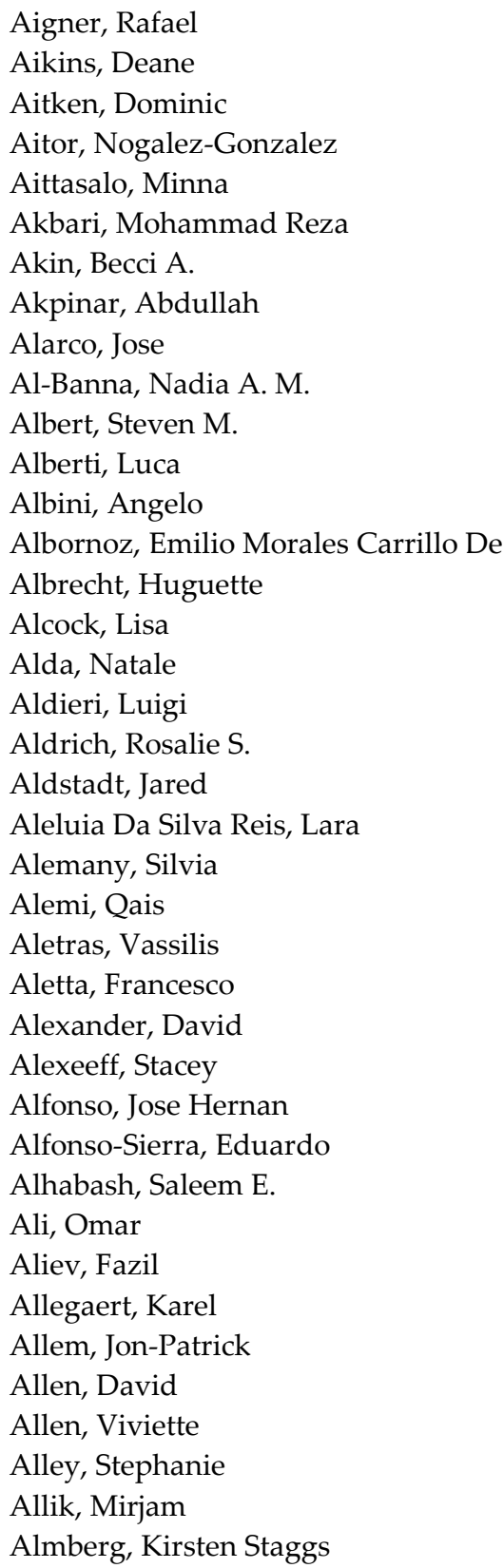


Almeida, Maria Adelaide Araújo

Almeida Palha, Joana

Alsharairi, Naser

Alshbool, Fatima Z.

Alves, Elisabete

Alves, Luis

Alves-Pereira, Mariana

Amagai, Teruyoshi

Amarasena, Najith

Amaro, Ana

Ambrey, Christopher

Ambrosino, Elena

Amer, Sherif

Amini, Reza

Amirkhanian, Yuri A.

Ammerman, Alice

Amor, Antonio J.

Amrith, Megha

Anaby, Dana

Anafi, Patricia

Anagnost, Susan E.

Ananga, Erick

Anastos, Kathryn

Andersen, Helle R.

Anderson, Britta L.

Andersson, Els-Marie

Andjelkovic, Mirjana

Ando, Takao

Andrade, Vanda

Andreassi, Maria Grazia

Andreeva, Elena

Andreoli, Roberta

Andreopoulou, Zacharoula

Andrew, Melissa

Andrews, Arthur

Anenberg, Susan

Angelidis, Apostolos

Angelone, Tommaso

Angermann, Jeff

Anifandis, George

Anna, Odone

Annunziata, Azzurra

Ansa, Benjamin

Antar, Alli

Anthony, Tracy

Antipova, Angela

Anton, Fernand

Antonanzas, Fernando

Antoñanzas, Fernando

Antonarakis, Gregory S.

Antosiewicz, Danuta Maria

Anttila, Ahti

Appell, Michael

Araki, Atsuko

Arakida, Mikako

Aranzabal, Asier

Archambault, Steven

Archibald, Daryll
Arcila Calderón, Carlos

Arcury, Thomas A.

Arenas Moreno, Alicia

Arias, Ana Victoria

Ariese, Freek

Armstrong, Pat

Arola, Antti

Arora, Amit

Arrandale, Victoria H.

Arrebola, Juan P.

Arreola, Sonya

Arthur, Frank

Arturo, Goldarazena

Asimakopoulos, Alexandros

Aslan, Asli

Aspray, Thomas

Assari, Shervin

Assimakopoulos, Vasiliki D.

Åström, Christofer

Atkinson, Sam

Atti, Anna Rita

Auger, Nathalie

Augsburger, Mareike

Auyeung, Tung Wai

Avellán, Tamara

Averill, Michelle

Avraam, Demetris

Aycock, Dawn

Ayonrinde, Oyekoya

Ayo-yusuf, Olalekan A.

Aysa-Lastra, Maria

Azeez, Adeboye

Aznar, Susana

Babatunde, Opeyemi

Babatunde, Emmanuel

Babu, Giridhara R.

Bacciu, Valentina

Baciu, Călin

Backer, Charlotte De

Backes, Todd

Badgley, Brian

Bađun, Marijana

Bae, Harold

Bahnfleth, William P.

Bahwere, Paluku

Baigorri, Roberto

Bailey, Kylie

Bailey, Catherine

Baird, Nicholas

Balachova, Tatiana N.

Balastegui, A.

Balawejder, Maciej

Balcazar, Hector G.

Balcom, Prentiss

Baldacchino, Frederic

Baldasano, Jose M.

Ballesteros, Mick

Balocco, Carla 
Balouch, Sara

Balter, Vincent

Balwicki, Łukasz

Ban, Masahito

Bana, Jinan

Banaitis, Audrius

Banerjee, Areen

Banik, Gouri Rani

Baragetti, Andrea

Barbara, Pancino

Barcelos, Anabela

Barclay, Linda

Barker, Bridget $\mathrm{M}$.

Barman, Apurba

Barnes-Josiah, Debora

Baron, Kelly

Bar-Or, David

Barranco Ruiz, Yaira

Barrington, Dani

Barron, Susan

Barros, Nilla

Barta, Csengele

Bartlett, Delwyn

Bartlo, Pamela

Bartoli, Francesco

Bartolucci, Giovanni Battista

Barton-Forbes, Michelle

Bartzis, John

Basile, Gianpaolo

Baska, Tibor

Basta, Nicholas Thomas

Basu, Pratyusha

Batey, Scott

Bathula, Chandra

Batista Henriques, Izabela

Battaglin, William A.

Baudry, Gino

Baugerud, Gunn Astrid

Baumgardner, Darrel

Baumgardner, Dennis J.

Baumgardt, Johanna

Baxter, Lisa

Bayat, Sahar

Bayer, Ilker S.

Bayer, Frank O.

Bazzani, Claudia

Bcheraoui, Charbel El

Beaty, Terri

Beaumont, Robin

Beauvais, Wendy A.

Beckman, Linda

Bednarczyk, Robert A.

Beery, Tom

Beil, Kurt

Beintner, Ina

Beizaee, Arash

Belan, Ingrid

Belay, Brook
BELIN De Chantemèle, Eric J.

Belknap, Robert

Bell, Simon

Bell-Anderon, Kim

Bellinger, David

Bellotti, Elisa

Bełtowski, Jerzy

Bend, Jack

Benitez Arciniega, Alejandra

Benjamin-Garner, Ruby

Bennett, James

Bennett, Julie

Benning, Jennifer

Bentley, Gillian

Bento, Douglas Mayer

Berardino, Santino

Berchialla, Paola

Bergamaschi, Enrico

Bergen, Doris

Berger, Matthias

Berger, Ann M.

Bergeron, Kim

Berglund, Erik

Bergouignan, Audrey

Berkhof, Johannes

Berkowsky, Ronald

Berland, Adam

Berli, Corina

Berliner, Elizabeth

Berman, Tamar

Bernabò, Nicola

Bernardi, Giulio

Bernardini, Giulia

Bernstein, Eve R.

Berrington, Janet

Berry, Meredith

Berry, Peter

Berry, William R.

Berry, Narelle

Berry, Tanya

Bertocci, Michele

Bertoni, Giuseppe

Bertram, Christine

Bertrand, Jane

Besenyi, Gina

Betz, Marian

Beute, Femke

Beutell, Nicholas J.

Bezner, Janet

Bhalla, Nikhil

Bhatta, Rhea

Bhaumik, Anusarka

Bhowmik, Arnab

Bhuiyan, Azad

Biagi, Federico

Bian, Junsong

Bianchi, Anna Maria

Bianchi, Fabrizio 
Bias, Thomas

Bibbo, Jessica

Bickham, David S.

Bidasee, Keshore

Biddle, Stuart

Bidmead, Elaine

Bierens, Joost

Biernasiuk, Anna

Bihuniak, Jessica D.

Bilgin, Aysenur

Billiard, Michel

Bin, Yu Sun

Bind, Marie-Abèle

Binfet, John Tyler

Bingham, Daniel

Binns, John

Binyamin, Jacqueline

Birch, Stephen (Steve)

Birch, Brian

Bisbal, Catherine

Bischoff, Karyn

Bisharat, Naiel

Biziuk, Marek

Black, Leon

Black, Carla

Blackall, Patrick

Blackmore, Carina

Blackmore, Chris

Bláha, Ladislav

Blair, Peter

Blanchette, Melanie

Blaschke, Paul

Blažíčková, Stanislava

Bleiweiss, Max

Block, Daniel

Block, Martin E.

Blukacz-Richards, E. Agnes

Blum, Igor $\mathrm{R}$

Blumer-Schuettea, Sara E.

Blüml, Victor

Boan, David

Board, Mary

Boardman, Jed

Boaz, Mona

Böckmann, Melanie

Bodin, Johanna

Bodin, Theo

Bogdanovica, Ilze

Bogunovic, Igor

Bojke, Chris

Bolton, James

Bondevik, Gunnar Tschudi

Bonetta, Silvia

Bonetta, Sara

Bongaerts, Brenda

Boniface, Sadie

Boomer, Jonathan S.

Boomhower, Judson
Boquien, Clair-yves

Borde, Theda

Borena, Wegene

Borisenkov, Mikhail F.

Bornman, Liza

Borras, Josep M.

Borrego, Carlos

Borrmann, Thomas

Borschmann, Rohan

Bortkiewicz, Alicja

Borzabadi-Farahani, Ali

Bos, Peter

Bos, Elske

Bosarge, Patrick L.

Boshuizen, Hendriek

Bosma, Hans

Bossew, Peter

Bossi, Giulia

Bosso, Luciano

Botella, Miguel C.

Bothe, Denise

Bouaïcha, Noureddine

Bouamra, Omar

Bourgeois, Denis M.

Bourliva, Anna

Bowatte, Gayan

Bowen, Deborah J.

Bower, Kelly M.

Boyas, Javier F.

Brackbill, Robert M.

Brahmbhatt, Tejal S.

Braks, Marieta

Branco, Manuel Castelo

Brands, Edwin

Brantsaeter, Anne Lise

Bratkic, Arne

Brattich, Erika

Bråtveit, Magne

Brauner-Otto, Sarah R.

Brčić Karačonji, Irena

Breitenfeld Granadeiro, Luiza

Brekke, Idunn

Bremberg, Sven

Breton, Mylaine

Brewer, George J.

Bridle-Fitzpatrick, Susan

Brien, James D.

Brimblecombe, Peter

Brinthaupt, Thomas M.

Britch, Seth Carroll

Britner, Preston A.

Brittain, Kelly

Broadbent, Jonathan M.

Brokamp, Cole

Bronzaft, Arline

Brookfield, Katherine

Brooks, Steve

Broom, Iain 
Broome, Kieran

Brough, Louise

Brown, Phil

Brown, Scott C.

Brown, David

Brown, Patrick

Brown, David M.

Brown, Richard J. P.

Brown, Julie

Brownstein, Naomi

Brunt, David

Bruschi, Fabrizio

Brusselaers, Nele

Brymer, Eric

Brzoska, Patrick

Bubici, Giovanni

Buchanich, Jeanine M.

Budde, Henning

Buitrago, María José

Bujnowska-Fedak, Maria Magdalena

Bunaciu, Rodica P.

Bungum, Timothy

Burden, Sandy

Burgess, John A.

Burgoine, Thomas

Burinskienè, Marija

Burkart, Katrin

Burke, Rita V.

Burke, Ronald J.

Burkhart, Craig G.

Burkowska-But, Aleksandra

Burlage, Robert

Burnett, Emma

Burris, Scott

Burrows, Stephanie

Burszta-Adamiak, Ewa

Burzynska, Monika

Bus, James S.

Busby, Chris

Buscarinu, Maria Chiara

Bush, Andrew

Busija, Lucy

Busjahn, Andreas

Butler, Matthew P.

Butler, John

Butner, Jonathan

Buttafuoco, Gabriele

Buttiglieri, Gianluigi

Buvik, Kristin

Byeon, Sang-Hoon

Byon, Ha Do

Byrne, Miriam

Bywaters, Paul

Cabello, Maria

Cabral-Pinto, Marina M. S.

Cabrerizo, Francisco Javier

Caccamo, Daniela

Cacciottolo, Mafalda
Caesens, Gaëtane

Caiado, Camila

Caiani, Enrico

Callahan, Damien L.

Callison, Kevin

Calogiuri, Giovanna

Calvo, Arlene

Calvo, Angela

Calvo, Concepción

Camacho, Luisa

Camacho-Miñano, Maria José

Camenisch, Todd D.

Camerino, Donatella

Camiciottoli, Gianna

Camille, Perchoux

Campa, A. M. Sa'nchez De La

Campana, Raffaella

Campbell, Frances A.

Campbell, Marilyn

Campbell, Corey

Campbell, Katarzyna Anna

Campbell Jenkins, Brenda W.

Campbell-Arvai, Victoria

Campo, Laura

Campobasso, Carlo P.

Camporeale, Rosalia

Candice, Young-Rojanschi

Cangussu Da Silva, Lucia Regina

Cantrell, Jennifer

Cao, Yong-Chang

Cao, Haiqun

Cao, Yongxiao

Cao, Gui-Ying

Cao, Guangyu

Capolongo, Stefano

Capolupo, Alessandra

Cappella, Joseph N.

Capra, Gian Franco

Caravanos, Jack

Cardemil, Esteban

Carey, Renee

Carignan, Courtney

Cariñanos, Paloma

Carling, Gregory T.

Carlisle, Gretchen K.

Carlo, Piero Di

Carlson, Jordan A.

Carlsson, Maria

Carlton, Jill

Carmine, Guarino

Caron, Rosemary

Caron, Jeffrey G.

Carr, Sam

Carr, Sherilene

Carré, Vincent

Carrero, Jose Antonio

Carrillo, Genny

Carroll, Suzanne J. 
Carson, Tiffany L.

Carta, Fabrizio

Cartalis, Constantinos

Carter, Wayne

Carter, Eric

Carugno, Michele

Caruso, Bethany

Caruso, Joseph A.

Carvalho, Anna C. C.

Carvalho, António Paulo

Casanovas, Carmen

Casas, Irene

Caserta, Donatella

Casey, Patricia

Caspi, Caitlin

Cassarino, Marica

Cassidy, Sophie

Cassidy, Laura

Castellanos, Paola

Castellazzi, Anna Maria

Castelli, Darla M.

Casteren, Jan Van

Castro, Shamyr Sulyvan

Cattani, Giorgio

Cavallini, Giorgio

Caxaj, Susana

Cayón De Las Cuevas, Joaquín

Cazzato, Eugenio

Cerchione, Roberto

Cerniglia, Luca

Cervellin, Gianfranco

César, Carina

Cetin, Mehmet

Cha, Christine B.

Chadborn, Neil

Chadwick, Philip

Chafe, Zoe Anna

Chai, Sangmi

Chai, Tian Feng

Chai, Xingyun

Chakraborty, Jayajit

Chalbot, Marie-Cecile G.

Chamine, Irina

Chan, Michael

Chan, Y. K.

Chan, Yu-Yi

Chan, Catherine B.

Chan, Alan H. S.

Chandran, Aruna

Chandrasiri, Upeksha

Chang, Chien-Chung

Chang, Po-Ju

Chang, Chih-Ching

Chang, Chen-Kang

Chang, Hsien-Yen

Chang, Kaowen

Chang, Kyu-Sik

Chang, Geng-Ruei
Chang, Hsien-Yen

Chang, Wen-Dien

Chang, Ta-Yuan

Chang, Shu-Man

Chao, How-Ran

Charisiadis, Pantelis

Charles, Luenda E.

Chatterjee, Avik

Chaves, Fabio

Che, Huizheng

Che, Weitao

Chelbi, Sonia T.

Chen, Jo-Hui

Chen, Jiangang

Chen, Chun

Chen, Yi-Min

Chen, Maosi

Chen, Shumei

Chen, Lv

Chen, Yi-Chun

Chen, Mei-Lan

Chen, HuaiH

Chen, Wenhao

Chen, Bo

Chen, Zhuo

Chen, Jui-Sheng

Chen, Yaoning

Chen, Xiang (Peter)

Chen, Kow-Tong

Chen, Joyce Chiao-Nan

Chen, Xiang

Chen, Weihong

Chen, Kai

Chen, Wei

Chen, Wei-Hsiang

Chen, Jeff Yi-fu

Chen, Tuo-Yu

Chen, Chehong

Cheng, Terence

Cheng, Kai-Wen

Cheng, An

Cheng, Evan

Cheong, Hae-kwan

Cherniack, Evan Paul

Cheung, Yin Ting

Chhatwal, Jugesh

Chi, Kai Hsien

Chiabai, Aline

Chiavegatto Filho, Alexandre

Chiefari, Eusebio

Chien, Chun-Ru

Chien, Yi-Wen

Chih, Ming-Yuan

Childs, Emma

Chileshe, Nicholas

Chillón, Palma

Chin, Cheng Siong

Chiou, Tzeon-Jye 
Chiou, Yuan-Yow

Chipps, Jennifer

Chisholm, June

Chitchumroonchokchai, Chureeporn

Chiu, Yu-Ling

Chiu, Yung-ho

Chiu, Chung-Yi

Chiva-Blanch, Gemma

Chmielowski, Krzysztof

Chmurzyńska, Agata

Cho, Clare

Cho, Do Yeon

Cho, Mihye

Choate, Peter W.

Chobot, Vladimir

Choi, Kelvin

Choi, Edmond

Choi, Young Min

Choi, Hyun-Deok

Choi, Jung-Seok

Chong, Marc

Chow, Bik Chu

Chrastný, Vladislav

Chrisinger, Benjamin

Christiansen, Hanna

Christoph, Rupprecht

Christophe, Waterlot

Chrzanowski, Łukasz

Chu, Chun-Hung

$\mathrm{Chu}$, Chunli

Chua, Tze-Ern

Chuang, Ting-Wu

Chuang, Hung-Yi

Chuang, Shu-Chun

Chuang, Kai-Jen

Chuang, Li-Min

Chuang, Wen-Ching

Chui, Helena

Chung, Hsin-Fang

Ciccacci, Cinzia

Ciccone, Marco Matteo

Ciclitira, Paul

Cidro, Jaime

Cieplak, Maciej

Ciesielczuk, Tomasz

Cihacek, Larry

Cilluffo, Giovanna

Cipollina, Maria

Cissé, Guéladio

Claassen, Liesbeth

Clair, Amy

Clark, Gary

Clark, Thomas A.

Claßen, Thomas

Clayton, Nicola

Cleland, Verity

Clements-Croome, Derek

Climie, Rachel
Clivot, Hugues

Close, Dan M.

Coelho, Luis Miguel Serra

Coetzer, Theresa

Cohen, Aaron

Cohen, Harold C.

Cole, Roy

Coleman, Mary

Coleman, William B.

Colicino, Elena

Colleran, Heather L.

Collings, Paul

Collins, Samuel

Collins, LaPorchia

Colombo, Claudio

Colson, Gregory

Comans, Tracy A.

Commodore, Adwoa

Comte, Katia

Conceição, Eusébio Z. E.

Concha, Jeannie

Connelly, Luke

Conrad, Zach

Conrad, Amy

Conte, Annamaria

Conti, Sara

Contini, Daniele

Contini, Daniele

Contzen, Nadja

Convertino, Matteo

Cooke, Barbara

Cooper, Kay

Cooper, Simon

Cope, Anna Barry

Cope, Michael

Cori, Liliana

Cornish, Stephen

Correa, Maria Elvira

Correa Marques, Rejane

Correa-Burrows, Paulina

Corsi, Daniel J.

Cortés-Castell, Ernesto

Cortese, Daniel K.

Cosco, Theodore D

Cosma, Alina

Costa, Nuno Manuel Sessarego Marques Da

Costa, Paul

Cote, Pierre-Olivier

Cotton, Robin T.

Coulson, Guy

Coulter, John

Coussens, Scott

Cova, Thomas

Cox, Georgina

Coxson, Pamela

Coyne, Mark

Coyte, Peter

Crabbe, Helen 
Craig, Laura

Craig, Brett

Cramb, Susanna

Craparo, Giuseppe

Craven, Michael P.

Crema, Stefano

Criado-Alvarez, Juan Jose

Crisafi, Francesca

Critchley, Hugo D

Croft, Rodney J.

Crohn, Helen M.

Cronin, Aidan

Cronkite, Ruth C.

Crooks, James L.

Cross, Paul C.

Crotty Alexander, Laura

Crowley, Jennifer

Crusius, Jan

Cryderman, Diana

Csavina, Janae

Csiszar, Agnes

Cubadda, Francesco

Cubero, Francisco Javier

Cucciniello, Raffaele

Culler, Corinna

Cummins, Ian

Cunha, Angela

Cunningham, Solveig Argeseanu

Curl, Cynthia L.

Curl, Angela

Curran, Louise

Curtis, Denice

Čvorović, Jelena

Cycoń, Mariusz

Cyrus, Elena

D'Elia, Lanfranco

Da Silva Guerra, Danielle Regina

Dąbrowska, Jolanta

Dalessandri, Domenico

Daley, Peter

Dalmolin, Ricardo

Dalton, Alice $\mathrm{M}$.

D'Amico, Mario

Dancer, Stephanie

D'Andrade, Amy

Dang, Zhi

Danielak, Dorota

Daniell, William

Daniels, Kevin

Daniels, Judith

Danielson, Kirstie K.

Dannenberg, Andrew

Dantzler, Prentiss

Daoud, Nihaya

Dapprich, Peter

Das, Anshuman

Dasgupta, Shouro

Datta, Rupa
David, Annette

Davies, Anita A.

Davies, Emma

Davis, David E.

Davis, Claudia M.

Davis, James

Dawson, John

De Andrade, Mariza

De Blas, Maite

de Brito, Jorge

De Cock, Rozane

De Donato, Francesca

De Francesco, Edi

De Giglio, Osvalda

De Graaff, Fuusje

De Gruijl, Frank

De Jesus Felicio, José Augusto

De Jonge, Jan

De Kwaadsteniet, Leontien

De La Cruz Toledo, Elia

De La Torre, Gabriel G.

De Lourdes Pereira, Maria

De Oliva, Samara Urban

De Oliveira Fernandes, Eduardo

De Palma, Giuseppe

De Souza, Roger-Mark

Dear, Keith

Dearden, John C.

Dederichs, Anne Simone

Dee, Anne

Deeprose, Catherine

DeGrauw, Xinyao

DeGuzman, Pamela B.

Deierlein, Andrea

Deisenhammer, Eberhard

DeJarnett, Natasha

DeKluizenaar, Yvonne

Del Giudice, Rita

Del Re, Brunella

DeLauer, Verna

DeLellis, Nailya

Delgado, Jordi

Delgado, Melvin

Delisle, Hélène

Delmelle, Eric M.

Delmonico, Matthew

Delong, Gayle

Deng, Peng

Deng, Yong

Denham, Bryan E.

Denholm, Justin

Dening, Tom

Denny, Clark

Dentinho, Tomaz

Deo, Randhir

Depken, Diane

Desai, Miraj U.

Desai, Jigar 
DeSesso, John M.

Deth, Richard

Dettweiler, Ulrich

Deutsch, Jonathan

Devane, Megan L.

Devaney, Carmel

Devaraj, Aishwarya

Devilee, Jeroen

Dewan, Ashraf

DeWeese, Robin

Dewey, Cate

Dewhirst, Timothy

DeWitt, Jamie C.

Dey, Aditi

Deymier, Alix C.

Deziel, Nicole

Dhana, Klodian

Dhar, Paramita

Dhuffar, Manpreet

Di, Jiangli

Di Ciaula, Agostino

Di Cristo, Cristiana

Di Domenico, Enea Gino

Di Liegro, Italia

Di Maria, Francesco

Di Martino, Ferdinando

Di Palma, Luca

Dias, Sonia

Dias, Rolando C. S.

Diaz, Julio

Diaz, Duarte

Dickey, Bret

Diderichsen, Finn

Dieris-Hirche, Jan

Diffin, Janet

Difranza, Joseph R.

Diller, Kenneth

Dillon-Wallace, Julie A.

Dimova, Elitsa

Ding, Qiang

Ding, Tianbing

Dini, Luciana

Dinkel, Danae

Diraco, Giovanni

Dirk, Richter

Dirks, Kim

Diroll, Benjamin

Dizmen, Coskun

Djebou, Dagbegnon C. Sohoulande

Dluholucký, Svetozár

Do, Thuy

Dobson, Marnie

Dockery, Douglas

Dodds, W. Jean

Doerr, Celeste

Dolney, Timothy J.

Domek, Gretchen J.

Domingo, Rosario
Domingue, Michael

Donat Vargas, Carolina

Donati, Cristiana

Donatuto, Jamie

Dong, Zhaomin

Dong, Zhao

Donny, Eric C.

Donohue, Maura

Donovan, John E.

Donzelli, Gabriele

Dorea, Caetano C.

Dorosh, Andriy

Dorri, Mojtaba

Dorszewski, Piotr

Dortet, Laurent

Dos Santos, Eduardo Ribeiro

Doshi, Saumil

Dou, Zhicheng

Dougherty, Michael John

Downing, Steven

Doyle, Frank

Drago, Francesco

Drancourt, Michel

Drapeau, Christopher

Dreassi, Emanuela

Drews-Botsch, Carolyn

Driezen, Pete

Dritselis, Chris

Droste, Dirk

Droste, Nic

Drottz-Sjöberg, Britt-Marie

Drzazga, Zofia

Du, Shushan

Duan, Hanchen

Dubois, Ute

Duby, Zoe

Dueker, M. Elias

Dugue, Benoit

Dumbili, Emeka

Dummer, Trevor

Dunkle, Ruth E.

Dunne, Eileen M.

Dunstan, David

Duodu, Godfred

Dupras, Charles

Duquette, Pierre

Duran, Nelida

Durando, Paolo

Durand-Zaleski, Isabelle

Durkin, Anne E.

Duron, Jacquelynn

Duszynska, Wieslawa

Dwivedi, Dipankar

Dyche, Jeff

Dyer, Tom

Dyson, Yarneccia D.

Eastin, Matthew D.

Ebeling, Peter 
Ebersviller, Seth

Ebisu, Keita

Ebmeier, Klaus

Echeverri, Margarita

Eckel, Sandrah P.

Eckert, Stine

Eddyani, Miriam

Edgar, Dale

Edwards, Joshua R.

Eedunuri, Vijay Kumar

Efird, Jimmy

Egashira, Shinji

Egawa, Shinichi

Eguchi, Nawo

Ehlers, Lars Holger

Ehlis, Ann-Christine

Ehrich, Marion

Eichstaedt, Christina

Eid, Patricia

Eime, Rochelle M.

Eiroa-Orosa, Francisco Jose

Eisler, Karen

Ekblad, Mikael

Ekmekcioglu, Cem

Ekström, Eva Charlotte M.

Ekundayo, Olugbemiga

Ekúndayò, Olúgbémiga T.

Ekuni, Daisuke

Ekwue, Edwin

El Ghoch, Marwan

El Khalloufi, Fatima

El Shahawy, Omar

Eldridge, Alison L.

Eleftheriadis, Theodoros

Elgazzar, Reda

Eliecer Coto, Eliecer Coto

Elinder, Carl-Gustaf

Elk, Ronit

Elliott, Mark

Elliott, Lewis

Elliott, Michael B.

Elmenhorst, Eva-Maria

Elstad, Jon Ivar

Elvik, Rune

Emmanuel, Evens

Emond, Jennifer A.

Engberg, Sandra J.

Engler-Stringer, Rachel

Erazo, Marcia

Erbacher, Terri A.

Erbas, Bircan

Erikson, Keith

Eriksson, Charli

Eronen, Johanna

Errede, Dawn

Erreygers, Guido

Errington, Gail

Esmene, Shukru
Esparcia, Javier

Espinel-Ingroff, Ana

Espinosa, Hugo G.

Espinosa-Ortiz, Erika

Esposito, Ciro

Essen, Elisabeth Von

Estape, Estela S.

Evans, Mary

Evelyn, Talbott

Evens, Anne

Everett, Kevin

Everts-Panman, Chantal

Ewen, Heidi $\mathrm{H}$

Exadaktylos, Aristomenis

Ezedine, Bouhlel

Fabbricatore, Mariantonietta

Fabbricino, Massimiliano

Fabiano, Bruno

Fabregat, Azael

Factor-Litvak, Pam

Fairlie, Ian

Fajersztajn, Lais

Falandysz, Jerzy

Falciglia, Pietro P.

Falconer, Ian

Falk, Martin

Falkenstein, Michael

Falkinham, Joseph

Fan, Tijun

Fan, Chao

Fan, Wen-Hong

Fan, Bin

Fang, Zheng

Faraj, Joycelyn

Farber, Naomi B.

Farhi, Dolly

Farina, Nicolas

Farma, Jeffrey M.

Farooq, Muhammad

Farrell, Anne F.

Farrington, Tom

Fasano, Evelina

Fauchier, Angele C.

Faustini, Annunziata

Favas, Paulo

Fei, Andrew Chang-Young

Feingold, Beth J.

Feng, $\mathrm{Yu}$

Feng, Lei

Fenton, Sue

Fera, Marcello

Ferguson, Alesia

Fernandez, Elizabeth

Fernández, Julio Blanco

Fernández-Bustos, Juan Gregorio

Ferrara, Nicola

Ferrari, Ed

Ferrario, Marco M. 
Ferras, Luís Jorge Lima

Ferrero, Enrico

Ferro, Giancarlo Antonio

Fiaschi, Simone

Figueiredo, Rafael

Figwer, Jarosław

Filon, Francesca Larese

Fine, James Burke

Finka, Andrija

Finkel, Madelon

Finlay-Jones, Amy

Finn, Symma

Fiocchi, Serena

Fiorito, Silvana

Firstenberg, Michael

Fischer, Florian

Fischer, Gabriele

Fischl, Andrea F. R.

Fish, Julie

Fisher, Gwenith G.

Fitzgerald, Neil

Fitzgerald, Niamh

Fitzsimmons, Suzanne

Flanagan, Sara V.

Flapper, Simme Douwe

Flecha, Ramon

Fleischhacker, Sheila

Fleiter, Judy

Fleming, Richard K.

Fletcher, Barbara A. Swore

Florek, Ewa

Florence, Curtis S.

Floros, Georgios

Fluegge, Kyle

Flynn, Jennifer

Foletta, Victoria C.

Fonseca, Ana Sofia

Font, Guillermina

Fontanals, Núria

Foody, Mairéad

Foong, Rachel E.

Foos, Brenda

Foote, Julie

Ford, Dawn

Ford, Judy

Forjuoh, Samuel N.

Forst, Linda

Forward, Sonja

Foster, Tim

Foster, Lauren

Fouladkhah, Aliyar

Founda, Dimitra

Foust, Richard D.

Fox, Mary

Fox, Glen

Frade, João

Fragkos, Konstantinos

Fram, Maryah Stella
Francescutti, Louis

Francis, Gary

Francis, Joel

Franck, Ulrich

Frank, Janet C.

Frank, Kevin

Frank, Saša

Franklin, Peter

Franz, Eelco

Franzen, Dave

Franzese, Adriana

Frayne, Bruce

Frazer, Kate

Frederick, Armah

Freedman, Darcy A.

Freeman, Toby

Freeman, Shrirra

Freihaut, James D.

Frew, Paula M.

Frey, Laura

Fried, Eiko I.

Friedman, S. H.

Friedrich, Rainer

Friger, Michael

Fristedt, Sofi

Froehlich-Grobe, Katherine

Fróes, Carmen

Fröhlich, Eleonore

Frohne, Tina

Fronczyk, Joanna

Frugé, Andrew

Frumkin, Howard

Ftanou, Maria

Fu, Qiang

Fuchs, Judith A.

Fudge, Nina

Fuente, Vicenta

Fuentes-Bargues, José Luis

Fuertes, Elaine

Fujibe, Fumiaki

Fujii, Yasuhito

Fujita, Masayuki

Fuks, Kateryna

Fukuda, Yoshiharu

Fukuda, Misao

Fuller, Richard

Fulthorpe, Roberta

Fung, Jimmy C. H.

Furuhashi, Kazuki

Furuta, Michiko

Fuster, Héctor

Gabbay, Mark

Gabutti, Giovanni

Gadagbui, Bernard

Gadomski, Anne M.

Gaffo, Angelo L.

Galanis, Athanasios

Galante, Francesco 
Galinha, Catarina

Gallelli, Luca

Gallis, Christos

Gamaldo, Alyssa

Gamble, Donald S.

Gamet-Payrastre, Laurence

Gander, Jennifer

Gandre, Coralie

Gannon, Judie

Gao, Zhaohui

Gao, Xiangpeng

Gao, Meng

Garcia, Valerie

Garcia, João Nuno Pinto Miranda

Garcia, Javier

García, Fernando

Garcia Bravo, Andrea

García-Bermejo, Juan

García-Matamoros, Jorge Bedia

García-Pérez, Javier

Gardner, Paul D.

Gardner, Mark

Garfield, Sara

Gari, Merce

Garnham, Bridget

Gartner, Coral

Gary-Webb, Tiffany

Garza-Rodríguez, Iliana De La

Gasana, Janvier

Gašević, Danijela

Gasparatos, Dionisios

Gast, Rebecca

Gatersleben, Birgitta

Gay, Melvin

Gaynor, Keith

Gazzola, Patrizia

Gdula-Argasińska, Joanna

Ge, Erjia

$\mathrm{Ge}, \mathrm{Cui}$

Ge, Xiaodong

Gea, Oliveri Conti

Gebbie, Kristine

Gee, Sarah

Geidne, Susanna

Geipel, Gerhard

Geisler, Corinna

Gemar, Mason

Gennari, Francesca

Gennuso, Keith P.

Geores, Martha

Georgiadis, Patroklos

Gerard, F. Jeane

Gerger, Heike

Gerrits, Lasse

Gerry, Christopher

Ghaffar, Seyed

Ghahari, Setareh

Ghanouni, Alex
Ghinea, Razvan Ionut

Ghosh-Dastidar, Bonnie

Giaccone, Valerio

Giacomino, Agnese

Giammanco, Anna

Gianatsi, Myrsini

Gibb, Herman

Gibbs, Bernhard F.

Gibson, Laura

Giedych, Renata

Gifford, Sandra

Gilden, Robyn

Giles, Luisa V.

Giles, Audrey

Gilissen, Renske

Gill, Tiffany

Gill, Simone V.

Gillander-Gådin, Katja

Gillespie, Duncan

Gilliver, Megan

Gilmour, Stuart

Gingrich, Chris

Giorgi, Gabriele

Giovene Di Girasole, Eleonora

Giraldo, Faber

Gisbert, Javier P.

Gitau, Margaret

Giuliani, Diego

Giuliano, Marina

Głąbska, Dominika

Glass, Joseph

Glicksman, Allen

Glynn, Peter J.

Gnambs, Timo

Gobbi, Erica

Gobejishvili, Leila

Godar, Dianne E.

Goettel, Mark

Gogos, Charalambos

Goicoa, Tomás

Golasi, Iacopo

Gold, Laura S.

Goldstein, Adam O.

Goletti, Delia

Golicnik, Barbara

Golla, Vijay

Goltz, Douglas M.

Gombac, Valentina

Gomes, Ana P.

Gómez Barrios, Xiomar

Gómez Piqueras, Pedro

Gómez-Bezares, Fernando

Gómez-Losada, Alvaro

Gonçalves, Luzia

Gong, Yang

Gonzalez-Barcala, Francisco Javier

Gonzalez-Casanova, Ines

Goodman, Julie 
Goodman, Pat

Goodwin, Michaela

Goodwin, Denise

Goodwin, Kelly D.

Goodwin, Ian

Gopalakrishnan, Bhaskaran

Gopal-Kothandapani, Jaya Sujatha

Gorczynski, Paul

Gordon, Ross

Gordon, Derrick M.

Görlich, Dennis

Gou, Zhonghua

Goulas, Antonis

Goyal, Rajni

Graber, Judith

Grabich, Shannon

Grady, Sue

Graffelman, Jan

Granada, Camille E.

Grandner, Michael A.

Granja, Mónica

Grant, William

Grant Ludwig, Lisa

Grases, Felix

Grattan, Lynn M.

Graveling, Richard

Graves, Lee

Gray, Lesley

Gray, Deborah

Gray, Emilie

Gray, Andrew

Green, James A.

Green, Nathan

Greenberg, Arthur

Greenleaf, Arie T.

Greer, Anna E.

Greim, Helmut

Grekousis, George

Greppi, Anna

Grether-Beck, Susanne

Grieger, Jessica A.

Griffin, Bronwyn

Grifoni, Roberta Cocci

Grigsby-Toussaint, Diana S.

Grischek, Thomas

Grochans, Elzbieta

Groffik, Dorota

Grossman, Ellie

Grucza, Richard

Grunig, Gabriele

Grunseit, Anne

Grzybowska, Katarzyna

Grzybowska, Ewa

$\mathrm{Gu}$, Danan

$\mathrm{Gu}$, Yuanyuan

Guerriero, Massimo

Gugusheff, Jessica

Guilcher, Sara
Guillén, J.

Guina, Jeffrey

Guitton, Matthieu J.

Gulati, Megha

Gulliver, Pauline

Gullón Tosio, Pedro

Gulson, Brian

Gumber, Anil

Gumbo, Jabulani R.

Gun, Richard

Gundogdu, Ozan

Gunier, Bob

Gunnarsdottir, Ingibjorg

Guo, Yu

Guo, Chun Xiang

Gusiatin, Mariusz

Gustafson, Alison

Gustafsson, Peik

Guthrie, Robert

Gutierrez, Kristie

Gütschow, Johannes

Guzek, Dominika

Guziana, Bozena

Guzzi, Gianpaolo

Gyorkos, Christina

Ha, Hoehun

Ha, Sandie

Haapala, Eero A.

Haarbauer-Krupa, Juliet

Haase, Hajo

Haboubi, Nadim

Habuda-Stanic, Mirna

Hachiya, Tsuyoshi

Hackett, Ruth

Hadimani, Ravi L.

Hadjikakou, Sotiris K.

Hagan, Christopher

Hagen-Zanker, Alex

Hagiwara, Nao

Hagner-Derengowska, Magdalena

Haile, Tadele Measho

Hailer, M. Katie

Hajek, André

Hajj, Dana

Hakulinen, Christian

Hales, Heidi

Halik, Ümüt

Hall, Nathaniel

Hall, Michael

Hall, Sophie

Hall, Ralph

Hall, H. Irene

Hall-Clark, Brittany

Halonen, Jaana I.

Halsall, Jamie

Halsband, Claudia

Haluza, Daniela

Hamann, Martine 
Hamano, Tsuyoshi

Hamat, Rukman Awang

Hamer, Gabriel

Hametner, Bernhard

Hamilton, David

Hamilton-Williams, Emma

Han, Young-Ji

Han, Dong Wook

Han, Xuemei

Han, Gang

Han, Qifeng

Han, Seung Jin

Han, Seung-Yong

Han, Bing

Haney, Jennifer

Hang, Xiaoshuai

Hanke, Wojciech

Hanley, Janet

Hanly, Paul A.

Hansen, Margaret

Hansen, Alana

Hansen, Anders Blædel Gottlieb

Haque, Reina

Haque, Saira

Harada, Kazuhiro

Harada, Kouji H.

Harbison, Justin

Harding, Ian

Hariharan, Harry

Haring, Robin

Harlan, Sharon

Harrell, Paul

Harrington, Kathleen F.

Harris, Allyssa L.

Harris, J. Irene

Harris, Keith

Harrison, Sayward E.

Harry, Kambezidis

Harstad, Ingunn

Hart, Joy L.

Harting, Janneke

Hartmann, James X.

Harvey, Carol

Harvey, Adam

Harville, Emily

Hashimoto, Naoto

Hassan, Rohail

Hatfield, Jerry

Hattaf, Khalid

Hatzopoulos, Stavros

Hatzopoulou, Marianne

Hauser, Peter

Hawcroft, Claire

Hawes, Martha

Hawkins, Roxanne D.

Hawkins, Keely

Hawkins, Marquis

Hawley, Brie
Hayami, Hitoshi

Hayes, Richard

Hayman, Laura L.

Haynes-Maslow, Lindsey

Hayward, R. David

Hazelton, Pamela

He, Daihai

$\mathrm{He}, \mathrm{Yi}$

He, Hongxuan

He, Guojian

He, Qi-Qiang

He, Xiaoqing

He, Qiang

Healy, Kristen

Heaton, Matthew J.

Heffelfinger, James

Heffner, Ashley Lewis

Heft, Harry

Hegerl, Ulrich

Heidmann, Iris

Heinen, Mirjam

Heinonen-Tanski, Helvi

Hejmanowski, Ryszard

Helbich, Marco

Hellwig, Michael

Helm, Paul

Hemphill, Jean Croce

Henderson, Barron

Hendricks, Joan C.

Hendriksz, Christian J.

Hendryx, Michael

Henning, Johannes I. F.

Henninger, William R.

Henning-Smith, Carrie

Henrickson, Mark

Henry, Tiernan

Henry, Kevin

Herbert, Rob

Herlache-Pretzer, Ellen

Hernández, Francisca

Herr, Raphael M.

Herranz, Raul Cano

Herrera, Ronald

Herrera, Manuel

Herrera-Melián, José Alberto

Herrmann, Alina

Herrmann, Fabian

Herzog, Karin

Herzog, Harold

Hess, Catherine Ann

Heßling, Martin

Hidaka, Brandon $\mathrm{H}$.

Higginbotham, Nick

Hiilamo, Heikki

Hiller, Susan

Hillier, Amy

Hilton, Claudia

Himmelstein, Mary 
Himmerich, Hubertus

Hinojosa, Melanie Sberna

Hinwood, Andrea

Hinyard, Leslie J.

Hirai, Itaru

Hiraishi, Akira

Hirata, Akimasa

Hirt, Edward R.

Hirvensalo, Mirja

Hiscock, Rosemary

Hitchings, Russell

Hjorth, Peder

Ho, Roger Chun-Man

Ho, ChinYu

Hoagwood, Kimberly

Hoare, Erin

Hock, Emma

Hodge, Allison

Hodgson, Ian

Hoekstra, Trynke

Hoepner, Lori

Hoffer Gittell, Jody

Hoffmann, Falk

Hofman, Jelle

Hogan, Anthony

Hogg-Johnson, Sheilah

Hollar, David W.

Holle, Rolf

Holloway, Ian W.

Holloway, Alison C.

Holzgraefe, Bernhard

Honda, Akiko

Hondula, David

Hong, Peiying

Hong, Young-Seoub

Hönig, Václav

Hoover, Joseph H.

Hori, Yuuichi

Hori, Hajime

Horiuchi, Masahiro

Horiuchi, Satoshi

Horst, Jeremy

Horton, Teresa H.

Horton, Graeme

Horvai, George

Hoshiko, Sumi

Hossein-Nezhad, Arash

Houghton, KM

Hovmand, Peter S.

Howard, Annie Green

Howard Wilsher, Stephanie

Howarth, Michelle

Howell, Andrew J.

Hribar, Lawrence

Hseu, Zeng-Yei

Hsia, Shih-Min

Hsieh, Cheng-Hsiung

Hsieh, Yi-Ping
Hsu, Hui-Chuan

Hsu, Stephen

Hsu, Hui-Chuan

Hsu, Minna

Hsu, Ming-Fu

$\mathrm{Hu}$, Hui

$\mathrm{Hu}$, Guoqing

$\mathrm{Hu}, \mathrm{Bo}$

$\mathrm{Hu}$, Zhiyong

Huang, Yu-Tung

Huang, Chao

Huang, Yu-Fang

Huang, Wu-Jang

Huang, Hung-Chung

Huang, Hao

Huang, Guangqun

Huang, Zhongwei

Huang, Yikun

Huang, Xiao

Huber, Jörg

Huettmann, Falk

Hughey, Morgan

Huguet, Nathalie

Hui, Xuan

Hülür, Gizem

Hume, Kelly R.

Hummel, Karin

Hung, Pei-Yin

Hung, Ivan F. N.

Hung, Yung-Tse

Hung, Mei-Chuan

Hung, Galen Chin-Lun

Hunt, Pamela

Hunt, Mercedes

Hunter, Ruth

Hursthouse, Andrew

Hurtig Wennlöf, Anita

Husáková, Lenka

Huss, Fredrik

Hutchinson, Jayne

Hutton, Vicki

Hyde, Melissa

Ian, Pagano

Ianieri, Adriana

Ickes, Melinda

Idler, Christine

Ifelebuegu, Augustine

Igic, Ivana

Ignatowicz, Katarzyna

Iguchi, Taisen

Ihle, Andreas

Iijima, Shigeo

Imeson, Anton

Imperiale, Davide

Infante-Rivard, Claire

Ingusci, Emanuela

Inniss, Enos C.

Innocent, Giles 
Inohara, Takehiro

Inoue, Ikuo

Inoue, Ken-ichiro

Ioka, Seiichiro

Iqbal, Tariq $\mathrm{H}$.

Iryo-Asano, Miho

Isaacson, Michal

Isani, Gloria

Isgor, Zeynep

Isidoro, Jorge M. G. P.

Islam, Mir Rabiul

Islam, Rafiq

Ismail, Ahmed

Istvan, Hernadi

Ittenbach, Richard F.

Iuliano, Sandra

Ivanov, Alexander V.

Ivey, Cesunica

Ivey, Cesunica E.

Iwahori, Toshiyuki

Izenstark, Dina Marie

Izzo, Viviana

J. Bidwell, Amy

Jaacks, Lindsay

Jackson, Sara

Jackson, Nicki

Jacobs, David

Jacobs, David E.

Jacobsen, Ramune

Jacobson, Sandra

JafariNasabian, Pegah

Jager-Hyman, Shari

Jagnoor, Jagnoor

Jahns, Lisa

Jaine, Richard

Jaki, Birgit

Jakovljevic, Mihajlo

Jalava, Katri

James, Andrew

James, Shirley A.

James-Todd, Tamarra

Janczarek, Monica

Jandl, Robert

Jang, Siwon

Jankowska, Marta

Janssen, Sabine

Jaradat, Abdullah

Jasienska, Grazyna

Jaśkiewicz, Michał

Jatoi, Ismail

Javed, Arshad Ali

Jay, Sarah M.

Jayawardene, Wasantha P.

Jazcilevich, Arón

Jego, Maeva

JENA, PRASANT

Jenkins, Jill A.

Jenkins, Rachel
Jennings, Wesley G.

Jennings, Mary Beth

Jennings, Charles R.

Jensen, Roger

Jensen, Marina Bergen

Jensen, Hanne Irene

Jeong, Bongju

Jeronimus, Bertus F.

Jia, Yunyan Andrea

Jia, Lili

Jiang, Shan

Jiang, Heng (Jason)

Jiang, Chengsheng

Jiao, Mingli

Jiao, Junfeng

Jiao, Tifeng

Jicha, Gregory A.

Jie, Cui

Jim, Luk

Jiménez, Lucía

Jiménez, Paul

Jiménez Rodríguez, M. Lourdes

Jiménez-Ballesta, Raimundo

Jimenez-Cisneros, Blanca

Jin, Yuchang

Jin, Qinhan

Jin, Jianjun

jin, yuan

Jin, Andrew

Jin, Li

Jindal, Charulata

Jiru, Mintesinot

Jo, Young Suk

Johnson, Nicholas

Johnson, Amy

Johnson, Evan C.

Johnson, Natalie

Johnson, Amy K.

Johnson, Dayna

Johnston, Jill

Jones, Antwan

Jones, Rena R.

Jones, Benjamin

Jones, Andrew

Jones, Peter

Jones, Rodney P.

Jones, Carwyn

Jones, Martin

Jonk, Yvonne

Jorens, Philippe G.

Jørgensen, Rikke

Joseph, Djenaba A.

Joshi, Peter

Joubert, Michael

Ju, Xiangqun

Juárez, Isaac Leobardo Sánchez

Julian, Tim

Jun, Sunghae 


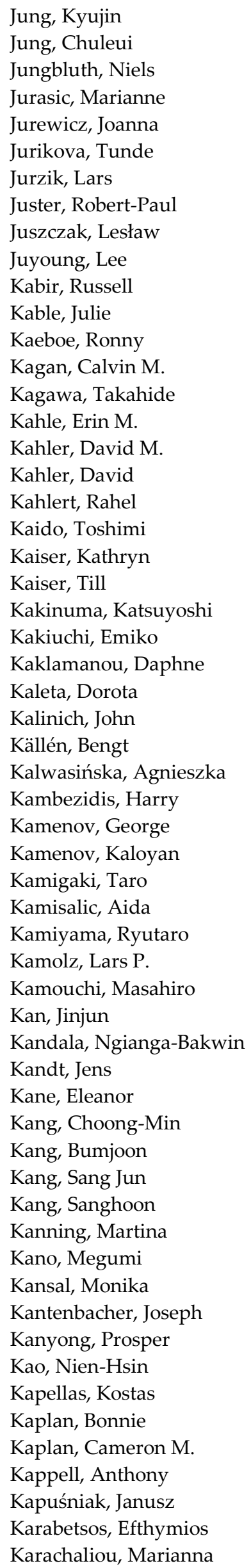

Karakitsios, Spyros

Karambelas, Alexandra

Karanis, Panagiotis

Karim, Salim Abdool

Karimli, Leyla

Kärkkäinen, Sirpa

Karlin, Bradley

Karpyn, Allison

Karriker-Jaffe, Katherine

Kasai, Naoya

Kashima, Saori

Katarina Lilith Johansson, Hanna

Katsoyiannis, Athanasios

Kaucic, Massimiliano

Kauffman, Anne Kathryn Marie

Kauko, Leiviskä

Kaushal, Navin

Kavé, Gitit

Kawada, Manabu

Kawai, Vivian K.

Keddell, Emily

Keegan, Thomas

Keida, Elixabeth

Keller, Joshua P.

Keller, Peter M.

Keller, Anita C.

Kellesarian, Sergio

Kelley, Dannielle

Kelley, Rachael

Kelly, Gabrielle

Kelly, Martina

Kelly, Inas

Kelly, Cheryl M.

Kelly, Kevin M.

Kemper, Han C. G.

Kempson, Ivan M.

Kenkel, Donald

Kennair, Leif Edward Ottesen

Kennedy, Jonathan

Kennel, Julie

Kenney, Christine

Kent, Jennifer

Kepka, Deanna Lee

Keraminiyage, Kaushal

Kermanshah, Amirhassan

Kershaw, Kiarri N.

Kersten, Ellen E.

Kersten, Jan Felix

Khalaf, Walaa

Khalil, Hussein

Khalil, Christian

Khamisa, Natasha

Khan, Izhar

Khan, Jahangir A. M.

Khanna, Sunali

Khanna, Savita

Khayesi, Meleckidzedeck

Khlebnikov, Andrei I. 
Khodadoust, Amid P.

Khubchandani, Jagdish

Khuder, Sadik A.

Khurram, Syed

Kian, Rozita

Kidd, Lisa

Kiesel, Lisa

Kietrys, David M.

Kilian, Reinhold

Kim, Harris Hyun-soo

Kim, Jay

Kim, Kyung-su

Kim, Minchul

Kim, BoRin

Kim, Dohyeong

Kim, Jinoh

Kim, Cheol-Ho

Kim, Woo-Yang

Kim, Min Joo

Kim, SoJung

Kim, Sun-Young

Kim, Woo Jin

Kim, Hyoung-Ryoul

Kim, Jong In

Kim, Seongho

Kim, Hyeong Su

Kim, Eun-Jin

Kim, Hyun-Woo

Kim, Hyun

Kim, Jinki

Kim, Young-jae

Kim, Seong Dae

Kimball, Samantha M.

Kimberly, Kelly

Kinchin, Irina

King, Tanya

King, Bryn

King, Marco-Felipe

Kinsky, Suzanne

Kintziger, Kristina

Kirchengast, Sylvia

Kirilenko, Andrei

Kirkham, M. B.

Kirkpatrick, Sharon

Kirnan, Jean

Kirtley, Olivia

Kita, Toshiko

Kivistö, Rauni

Kjellgren, Anette

Kjellstrom, Tord

Klaperski, Sandra

Klarin, Bengt

Klaschka, Ursula

Klaus, Schäfer

Kleiman, Evan M.

Klein, Irwin

Klika, Riggs

Klimes-Dougan, Bonnie
Klimkowicz-Pawlas, Agnieszka

Kluczniok, Katharina

Knapp, Caprice A.

Knappett, Peter

Knapstad, Marit

Kneil, Kali

Knight, John

Knott, Rachel

Knox, Oliver

Knudsen, Gabriel Arther

Kobayashi, Natsuko I.

Koch, Manfred

Kociu, Arben

Kocjančič, Tina

Kocot, Joanna

Koda, Eugeniusz

Koduru, Janardhan Reddy

Koelmans, Bart

Kohlitz, Jeremy

Kohno, Kunie

Koizumi, Hiroyasu

Kok, Gerjo

Kolar, Mila

Kolmes, Steven A.

Kõlves, Kairi

Komljenovic, Dragan

Konarski, Jan

Kondo, Michelle

Kong, In-soo

Kongar, Elif

Konradsen, Hanne

Kontogiorgis, Christos

Koo, Chamun

Koppe, Janna G.

Korajkic, Asja

Koreanschi, Andreea

Korhonen, Ilkka

Korkeila, Jyrki

Kormas, Konstantinos

Kornhaber, Rachel

Korzeniewska, Ewa

Kos, Bor

Kósa, Karolina

Kosarac, Ivana

Koser, Khalid

Kosmider, Leon

Kosova, Klara

Kotula-Balak, Malgorzata

Kousha, Termeh

Kovacic, Melinda Butsch

Kovanen, Petri T.

Kow, Alfred Wei Chieh

Kowalska, Małgorzata

Kowalska-Góralska, Monika

Koyama, Asuka

Koyama, Yu

Kozisek, Frantisek

Kraak, Vivica 
Kraft, Michael

Krakow, Melinda

Kral, Michael

Krata, Agnieszka Anna

Kraut, Allen

Krawczyk, Magdalena

Krienert, Jessie L.

Krist, Lilian

Kristiansen, Jesper

Kristjansson, Alfgeir

Kroon, Jeroen

Kroon, Frederieke J.

Krüger, Fred

Krukowski, Henryk

Krupinski, Elizabeth A.

Krushkal, Julia S.

Krysinska, Karolina

Kuang, Ze-Min

Kucharska-Newton, Anna

Kudielka, Brigitte M.

Kudláček, Michal

Kudłak, Błażej

Kudumija Slijepčević, Marija

Kuhfuss, Laure

Kuhnlein, Harriet V.

Kulaga, Zbigniew

Kumanyika, Shiriki K.

Kumar, Vikas

Kumar, Ratika

Kumar, Binod

Kumar, Krishan

Kundi, Michael

Kunert, Karl

Kunst, Anton

Kuo, Shu-lung

Kurade, Mayur B.

Kurti, Allison N.

Kuwahara, Keisuke

Kvaal, Kari

Kwak, Moon K.

Kwak, Kyung-Hwan

Kwan, Mei-Po

Kwok, Richard

kwok-Wing, Chau

Kyne, Dean

Kypriotakis, George

Kyung, Gyouhyung

Kyzas, George Z.

L. Schmitz, Cathryne

Lacaze, Bernard

Ladeira, Carina

Låftman, Sara Brolin

Lagroye, Isabelle

Lahiri, Sriyanka

Lai, Julian C. L.

Lai, Chia-Hsiang

Lai, Ka Man

Laidlaw, Mark
Laird, Brian

Lake, Iain

Lallier, Thomas E.

Lam, Tsan Yuk

Lam, Simon C.

Lam, Sean Shao Wei

Lang, Ingeborg

Langaas, Sindre

Langer, Sarker

Langiano, Elisa

Lanning, Beth A.

Lanou, Amy Joy

Lansley, Guy

Lantes, Oscar

Lao, Chunhuan

Large, Matthew

Larkins, Nicholas

Larsen, Mark

Larson, Lincoln

Larson, Brianna

Lassnig, Heimo

László, Makra

Lauerma, Hannu

Lauridsen, Jørgen Trankjær

Lauritano, Dorina

Laverack, Glenn

Lavigne, Eric

Lavin, Roberta

Lavkulich, Les M.

Lavoie, Raphael A.

Lavoie, Kim

Law, Jane

Law, Evelyn

Ławniczek-Wałczyk, Anna

Lawrence, Claire

Lawrence, David A.

Lawrence, Wanda

Lawrenson, Ross

Laws, Michael Barton

Lawson, Joshua A.

Lawson, Justin

Laxe, Sara

Lazarin, Angélica

Lazzerini, Marzia

Le, Huong

Le, Cuong Nguyen

Leach, Heather

Leader, Avi

Leary, Emily

Leavy, Justine

Lebeck, Janne

Lebel, Alexandre

Leboeuf, Gael

LeClair, Mark S.

Ledda, Mario

Lee, Joe-Ming

Lee, Young Ji

Lee, Jongho 
Lee, Eunsu

Lee, Eul-Bum

Lee, Paul H.

Lee, Regina L. T.

Lee, Joseph

Lee, Nora L

Lee, Jae Eun

Lee, Gyu-Cheol

Lee, Joon

Lee, Won Jin

Lee, Jin-Yong

Lee, Joseph G. L.

Lee, Jong Seon

Lee, Petrona

Lee, Jiyoung

Lee, Ho-Won

Lee, Wing-Kee

Lee, Jae Seung

Lee, Do-Gil

Lee, Kuo-Kau

Lee, Huei-Jane

LEE, Yiu Yin Raymond

Lee, Yung-Jaan

Lee, Kyung-Yil

Lees, Rosemary

Lefering, Rolf

Lefterov, Iliya

Legault, Laurent

Leggat, Sandra G.

Lehning, Amanda

Leibovici, Didier

Leidl, Reiner

Leiherer, Andreas

Leisner, Jørgen

Leisyte, Liudvika

Lekies, Kristi S

Lelard, Thierry

Lemke, Stefanie

Lenart-Boroń, Anna

Leng, Xiaoyan Iris

Lent, Michelle R.

León, Gerardo A.

León Pérez, Jose Maria

Leonard, Anne

Leone, Antonio

Leone, Matthew C.

Leong, David Tai

Leonhard, Christoph

Lépy, Élise

Lercher, Peter

Lerchl, Alexander

Lê-Scherban, Félice

Leung, Katherine

Leung, Janni

Levallois, Patrick

Levi-Belz, Yossi

Levizou, Efi

Lewinson, Terri
Lewis, Kathleen

Li, Zijian

Li, Weidong

Li, Dongmei

Li, Siwei

Li, Chenyu

Li, Dapeng

Li, Xuemei

Li, Sen

Li, Fuzhong

Li, Xiangyu (Dale)

Li, Yonghua

Li, Jianghong

Li, Yongjian

Li, Xuebing

Li, Xiujun

Li, Baoguang

Li, Shandy

Li, Ying

Li, Weijuan

Li, Rui

Li, Zoe

Lian, Tina

Lian, Ie-Bin

Liang, June

Liang, Yating

Liao, Haifeng

Liao, Yung

Lichand, Guilherme

Licitra, Gaetano

Liddle, Brantley

Lieberman, Lauren Joy

Liew, Zeyan

Liguori, Giorgio

Liller, Karen

Lim, Hyeyeun

Lim, Keah Ying

Lim, Megan

Lin, Wei

Lin, Thung-hong

Lin, Jin-Ding

Lin, Ye

Lin, Shengda

Lin, Ken-Yu

Lin, Pao-Hwa

Lin, Chiu-Yue

Lin, Yi-Chin

Lin, Jonqlan

Lin, Zhoumeng

Lin, Xiong

Lin, Pao-Hwa

Lin, Ming-Tai

Lin, Yan

Lindahl, Johanna

Lindert, Jutta

Lindholm, Dan

Lindong, Ian

Lindquist, Mark 
Lindsay, Ryan

Link, Denise

Liou, James

Lipnicki, Darren

Lisa, Antonella

Litescu, Simona Carmen

Litman, Todd

Little, Julian

Liu, Pei-Yang

Liu, Jia Coco

Liu, Li-Fan

Liu, Kevin Fong-Rey

Liu, Xin

Liu, Yu Wei

Liu, Cheng

Liu, Hui-Ming

Liu, Jie

Liu, Chiang

Liu, Barbara

Liu, Chiung-Ju

Liu, Yong

Liu, George

Liu, Chien-Tsai

Liu, Aijun

Liu, Zijuan

Liu, Guoxiang

Liu, Chun

Liu, Bin

Liu, Ko-Fei

Liu, Chengfang

Liu, Jingling

Liu, Hai-Ying

Liu, Yuwei

Liu, Zhaorong

Liu, Peide

Liu, Gang

Liverani, Silvia

Livingston, Mark

Livingston, Michael

Llorens, Jordi

Llorens, Eugenio

Lo, Huang-Mu

Loaiciga, Hugo A.

Lochbaum, Marc

Lockie, Robert

Loerbroks, Adrian

Logsdon, Sally

Löhmus, Mare

Lokkerbol, Joran

Lomonaco, Guglielmo

Lonardo, Amedeo

Long, Thomas B.

Longa, Claudia Maria Oliveira

Lopez, Nanette

Lopez Jornet, Pia

López López, Daniel

Lopez-Aparicio, Susana

López-Fernández, Macarena
López-Vizcaíno, Rubén

Loppi, Stefano

Lorber, Mateja

Lorber, Sophie

Loss, Julika

Loton, Dan

Lou, Song

Louis, Joseph

Loureiro, Ana

Loury, Sharon

Love, Tanzy

Lovreglio, Piero

Low, Jacqueline

Löytönen, Markku

Lozano-Sufrategui, Lorena

Lu, Guining

$\mathrm{Lu}, \mathrm{Yu}$

Lu, Yi

Lu, Binbin

Luan, Hui

Luben, Tom

Lucas, Paul L.

Lue, Fang

Lugg-Widger, Fiona

Lugo, Alessandra

Luick, Bret R.

Luís, Ana

Lukaszuk, Judith

Luna, Gian M.

Lund, Sandy

Lundälv, Jörgen

Lundeen, Elizabeth

Luo, Pingping

Luo, Xiao-San

Luo, Yan

Lusa, Merja

Lusk, Anne

Lyall, Kristen

Ma, Yiyi

Ma, Xiaoguang

Ma, Zheng Feei

Ma, Peng

$\mathrm{Ma}, \mathrm{Li}$

Ma, Guansheng

Mabuchi, Kiyohiko

MacArthur, Georgina

MacCarthy, Michael

Mach, Pavel

Machado, Carolina

Machell, Georgia

Macher, Janet M.

Mackinnon, Gillian

MacLeod, Catherine A.

MacMillan, Freya

MacNell, Lillian

Macy, Jonathan T.

Madonia, Alice

Madsen, Trine 
Maeda, Takafumi

Magee, Madeline

Magnani, Corrado

Magnavita, Nicola

Maguire-Jack, Katie

Mah, Catherine

Maharaj, Savi

Maher, Jaclyn P.

Maher, Christopher

Maher, William

Mahmud, Khan M.

Mai, Lu

Maicaneanu, Sanda Andrada

Maier, Birga

Main, Gill

Mainka, Anna

Maisha, Buuma

Majeed, Ban

Majewska, Maria Dorota

Majumdar, Indrajit

Maksymiuk, Gabriela

Malandrino, Mery

Malara, Alba

Malecki, Kristen

Maleknia, Simin D.

Malesios, Chrisovalantis

Malhotra, Praveen

Mali, Matilda

Malissiova, Eleni

Mallow, Ole

Malone, Susan K.

Mambretti, Stefano

Mamen, Asgeir

Mamudu, Hadii

Mamudu, Hadii M.

Manandhar, Rejina

Mancini, Francesco

Manderscheid, Ronald W.

Manikas, Andrew S.

Manjunath, Manubolu

Mann, Samuel Joseph

Mannucci, Pier Mannuccio

Mansourian, Ali

Manzo, Ciro

Mao, Liang

Marano, Nina

Marchant, David

Marchi, Michela

Marczylo, Emma

Margaritis, Lukas

Márialigeti, Károly

Mari-Dell'Olmo, MArc

Marin, Daniela

Marino, Joseph

Markevych, Iana

Marks, Ray

Marreilha Dos Santos, Ana Paula

Marrs, Carl
Marston, Louise

Martin, Michelle

Martin, Richard H.

Martin, Jeff

Martinez, Antonio J. Torija

Martínez, Ramón Peña

Martinez-Camblor, Pablo

Martinez-Colon, Michael

Martínez-Guitarte, José-luis

Martinez-Molina, Antonio

Martins, Maria Luisa Louro

Martins, Maria

Marzano, Lisa

Mascherini, Gabriele

Maslow, Joel N.

Mason, Lisa Reyes

Massaccesi, Luisa

Massas, Ioannis

Masten, Susan

Masterson, Erin

Masuda, Camlyn

Matanle, Peter

Mateo-Garcia, Monica

Matheson, Anna

Mathew, Supriya

Mathews, Anne E.

Mathur, Manu

Matsubara, Keiichi

Matsuda, Kazuhiko

Matsui, Kenji

Matsumoto, Ken-ichiro

Matud, María Pilar

Matz, Carlyn J.

Mauny, Frederic

Maupomé, Gerardo

Maurer-Fazio, Margaret

Mavaddat, Nahal

Mavromichalaki, Helen

Mawson, Anthony R.

Maxim, L. Daniel

Mayer, Adam

Maynard, Olivia M.

Maynard, Leah Michele

Mayner, Lidia

Mazumdar, Sumit

Mazur, Joanna

Mbulo, Lazarous

McBride, Devin W.

McCabe, Brian

McCaffrey, Nikki

McCann, Katharine

McCarthy, Valerie

McCormack, Lacey

McCormick, Sabrina

McCoy, Sarah J. Breese

Mccoy, Stephanie

McCreesh, Nicky

McCullugh, Cathy 
McDermott, Suzanne

Mcdonald, Ted

McEachan, Rosie

McElroy, Jane

McFadden, Jonathan

McGovern, Patricia M.

McGregor, Carolyn

McGuire, Sarah

McKay, Carly

McKeganey, Neil

McKendry, Corina

McKenzie, Erica

McKenzie, Lisa

McKenzie, Paul

McKinley, Jennifer

McKinley, Michael

McKinstry, Carol

McLaughlin, John E.

McLeod, Geraldine F. H.

McLeroy, Kenneth

McLuskey, John

McMorris, Terry

McQuade, Kevin J.

McQuaid, Christopher Finn

Meacham, Susan L.

Mears, Stephen A.

Meddings, David

Medina-Vera, Myriam

Meerlo, Peter

Mehmood, Amber

Mehta, Tapan

Mei, Chuansheng

Meijer, Eline

Melander-Wikman, Anita

Melendez, Rita

Melo Zurita, Maria De Lourdes

Meloni, Italo

Mendelsohn, Colin

Mendes, Ana Sofia Estevão

Mendes, Maria Paula

Méndez-Lázaro, Pablo A.

Mercer, Jenny

Mercille, Julien

Merget, Rolf

Merino, Gracia

Merklinger-Gruchala, Anna

Merrick, Teresa

Merry, Melissa

Methner, Ulrich

Meyer, Stephen

Meyer-Rochow, Victor Benno

Mézes, Miklós

Meziti, Alexandra

Mguni, Patience

Mi, Zhifu

Mian, Caterina

Michaelidou, Alexandra-Maria

Michailidis, Panagiotis
Michalke, Bernhard

Michelozzi, Paola

Michikawa, T.

Micu, Adrian

Middleton, Nick

Midgley, Adam

Mielgo Ayuso, Juan

Mielke, Howard

Mier, Nelda

Mignon, Sylvia

Mikkelsen, Bent Egberg

Mikkers, M. C.

Mildorf, Tomas

Milic, Natasa

Milici, Frances Fleming

Milla, Saajanaho

Miller, Judith S.

Miller, Charles

Miller, Emma R.

Miller, Shelly

Min, Leilei

Mingo, Antonio

Mínguez-Alarcón, Lidia

Minicozzi, Pamela

Mir, Debby

Miraglia, Mariella

Mirauda, Domenica

Mirlohi, Susan

Mirman, Jessica H

Miron, Isidro Juan

Misciagna, Giovanni

Mita, Damiano Gustavo

Mitchell, Joshua J.

Mitchell, Lana

Mitchell, Daniel

Mitchell, Rod

Mitkowski, Piotr

Mitrou, Francis

Miwa, Tomio

Miyamoto, Kuniaki

Miyoshi, Shin-ichi

Mizuno, Koh

Mizuno, Tooru

Mo, Lingfei

Mo, Jinhan

Mohatt, Nathaniel

Mohebbi, Mohammadreza

Möhner, Matthias

Moise, Imelda K.

Mokkenstorm, Jan

Mole, Richard

Moliner-Urdiales, Diego

Molino, Monica

Mollerup, Steen

Momtaz, Salim

Moncada, Stefano

Mondal, Debapriya

Mónica, Perez Bazán Laura 
Monroe, Kathy

Monroy, Fernando

Monsur, Muntazar

Montes, Agustín

Montgomery, JoLynn P.

Montllor, Joan

Montuori, Paolo

Monyeki, Kotsedi

Moon, Christopher J.

Mooney, Sandra

Moore, Simon C.

Moore, Paul

Moore, Geromy

Moore, Karen

Moosmann, Bernd

Mora, Rodrigo

Morales Suárez-Varela, María M.

Morawa, Eva

Moreno, Teresa

Morera, Maria

Morgan, Marsha

Morin, Cory

Morioka, Tomoaki

Morita, Manabu

Moriya, Shingo

Morphett, Kylie

Morrell, Holly E. R.

Morris, Cody

Morris, Melanie

Morris, Robert D.

Morrissey, Joanna

Morrone, Michele

Morrow, Jennifer

Moskalewicz, Jacek

Mosnier, Anne

Moss, Antony

Mouchtouri, Varvara A.

Mougiakakou, Stavroula

Moustris, Konstantinos

Moynier, Frédéric

Mozumder, Mohammad

Mpofu, Elias

Mrhálek, Tomáš

Mücke, Hans-Guido

Mudde, Aart

Mudge, Suzie

Mueller, Megan P.

Muggeo, Vito M. R.

Mui, Kwok-wai

Mulia, Nina

Müller, Jochen A.

Müller, K. W.

Müller, Johannes

Müller, Ruth

Mulligan, Hilda

Mulligan, Kate

Mullin, James M.

Mulvaney, Caroline A.
Mundorf, Chris

Mundorf, Christopher

Mundt, Sabine

Munir, Mariya

Munoz, Macarena

Murakami, Keiko

Muramatsu, Naoko

Murante, Anna Maria

Murbach, Manuel

Murff, Sharon

Murphy, Dominic

Murphy, Georgina A. V.

Murray, Megan

Murtaugh, Maureen

Musaad, Salma

Mushtaq, Nasir

Musk, Bill

Musliner, Katherine

Musmarra, Dino

Mutoh, Michihiro

Mutti, Luciano

Mydlarz, Charlie

Myers, Candice

Myrberg, Gunnar

$\mathrm{Na}$, Huimin

Nadais, Helena

Nagayoshi, Mako

Naha, Pratap C.

Nahar, Vinita

Nahar, Vinayak K.

Nakamura, Masashi

Nangia-Makker, Pratima

Nanjappa, Manjunatha

Nanos, Nikos

Nante, Nicola

Napiórkowska-Krzebietke, Agnieszka

Narasimhan, Sukanya

Nardell, Edward

Nash, Poppy

Nash, Sarah

Nassan, Feiby

Navacchia, Maria Luisa

Navarrete-Muñoz, Eva Maria

Navarro, Jose

Navarro Olivas, Raúl

Nawrocka, Agnieszka

Naylor, Lindsay

Naz, Sabrina

Nazeer, Majid

Nazir, Salman

Nduka, John

Nduna, Mzikazi

Negrón, Rosalyn

Nelson, Lindsay D.

Nemeth, Lynne

Nenna, Raffaella

Neslund-Dudas, Christine

Neto, Maria Augusta 
Neumann, Tim

Newall, Emma Rochelle

Newbold, K. Bruce

Newby, Ruth

Newman, John F.

Newman, Alan P.

Newton, John

Neymotin, Florence

$\mathrm{Ng}$, Chris Fook Sheng

$\mathrm{Ng}$, Lisa C.

$\mathrm{Ng}$, Isabella F. S.

$\mathrm{Ng}$, Kwok

Nguyen, Ruby H. N.

$\mathrm{Ni}$, Debing

Nicholas, Jennifer

Nicholls, Keith

Nichols, Michelle G.

Nie, Peng

Nieboer, Evert

Niebylski, Mark

Niedzielski, Przemysław

Niehoff, Jens-Uwe

Nielsen, Samara Joy

Niemann, Lars

Nieminen, Pentti

Nieves, Kourtney

Nijmeijer, Arian

Nikitas, Alexandros

Nikolaidou, Maria

Nobmann, Elizabeth D.

Nolan, Laura B.

Noli, Fotini

Nomura, Kyoko

Noonan, Robert

Noordam, Raymond

Nori-Sarma, Amrutasri

Norman, Trudy

Normanno, Giovanni

Norra, Christine

Norval, Mary

Noszticzius, Zoltán

Notley, Caitlin

Notredame, Charles-Edouard

Novack, Tessio

Novellino, Alessandro

Nowak, Glen

Nowak, Michal S.

Nowalk, Mary Patricia

Nowicka, Paulina

Nowicka, Grażyna

Nowotny, Norbert

Nshimyimana, Jean Pierre

Nübling, Rüdiger

Nüesch-Inderbinen, Magdalena

Nugraha, Adi

Núñez-Delgado, Avelino

Nwanodi, Oroma

Nyangweso, Mary
Nyholm, Maria

Nyrud, Anders Q.

O'Higgins Norman, James

$\mathrm{O}^{\prime}$ dwyer, Lisel

O'Hare, Liam

Oberbeckmann, Sonja

Oblad, Timothy

Obolewski, Krystian

O’Brien, Karel K.

Ochiai, Tsuyoshi

O'Connor, Richard

Octaria, Rany

Odland, Jon Øyvind

O'Dwyer, Jean

Oesterle, Sabrina

Ogeil, Rowan

Ogórek, Rafał

O'Hare, Anthony

Ohnishi, Masatoshi

Ohtsuru, Akira

O'Keefe, Denise

Okoro, Chinyere

Okuda, Masayuki

Okulicz-Kozaryn, Adam

Olafsdottir, Gunnthora

Olde Rikkert, Marcel G. M.

Oliveira, Teresa

Oliveira, Paula Alexandra Martins De

Oliveira, Cláudia Sirlene

Olivier, Jill

Olsen, Jonathan

Olu, Olushayo

Oluwole, Oluwafemi

Omoruyi, Felix

O'neill, Eoin

Onicescu, Georgiana

Opaas, Marianne

Orbea, Amaia

Orecchio, Santino

Ormandy, David

Ornelas, India

Ornelas, Yoshira

Orosa, José

Orpana, Heather

Ortega, Enrique

Ortega, John

Ortiz-Silla, Roque

Orton, Sophie

Orton, Elizabeth

Osborne, Nicholas

Osin, Evgeny N.

Osman, Alfatih A. A.

Osteen, Philip

Ostrowski, Lawrence E.

Ota, Atsuhiko

Otero, Marta

O'Toole, Tim

Otsuki, Takemi 
Otte, Dietmar

Ou, Judy

Ou, Yichang

Ou, Chunquan

Oudin, Anna

Ouyang, Jianming

Ovalle-Perandones, Maria Antonia

Oviedo Ocaña, Edgar Ricardo

Owusu, Andrew

Ozaki, Noriatsu

Ozawa, Mio

Ozbay, Gulnihal

Ozoe, Yoshihisa

Pääkkönen, Rauno

Paap, Kenneth R.

Paavilainen, Eija

Pacek, Lauren

Pachego-Torgal, Fernando

Padula, Amy

Paganotti, Giacomo Maria

Page, Kristen

Pai, Chi-Wei

Palagyi, Anna

Palazzolo, Dominic L.

Palmer, Jack A.

Palmer, Raymond F.

Palmisano, Giovanni

Palsamy, Periyasamy

Paludi, Michele

Pammolli, Andrea

Pampel, Fred

Pan, Meixia

Pan, $\mathrm{Xi}$

Pan, Zhujun

Pan, Jiayan

Pan, Ming-Ju

Panagiotidi, Maria

Panagiotopoulos, Panagiotis

Pang, Chan Siew

Pang, Myung-Geol

Pani, Shantanu Kumar

Panicucci Zíková, Alena

Pankratz, Curtis

Pant, Pallavi

Panza, Francesco

Papadakis, Raffaello

Papadimitriou Olivgeris, Matthaios

Papadimitriuou, Konstantinos

Papadopoulos, Nestoras

Papadopoulou, Chrissanthy

Papapostolou, Vasileios

Papazafiropoulou, Athanasia K.

Pape, Kristine

Pape, Hans-Christoph

Pappas, Richard Steven

Paquet, Catherine

Parajuli, Rajendra Prasad

Parajuli, Rajendra
Parazzini, Marta

Pardío-Sedas, Violeta T.

Parent, André Anne

Parise, Carol A.

Parisi, Mimmo

Parisi, Alfio

Park, Il-Kwon

Park, Subin

Park, Boyoung

Park, Sojung

Park, Jong-Ik

Park, Sook-Hyun

Park, Jae Bum

Park, Van

Parke, Adrian

Parker, Kimberly

Parker, Stacey

Parlesak, Alexandr

Parr, Evelyn

Parry, Sharon

Partonen, Timo

Pasanen, Tytti

Paschalidou, Anastasia K.

Paschall, Mallie

Pascual Aguilar, Juan Antonio

Pasini, Dario

Passannante, Marian

Pasti, Luisa

Pastor, Manuel

Patel, Kushal

Patel, Neela K.

Pathak, Ram D.

Patton, Allison

Paudel, Shishir

Paull, Sara

Paulsen, Peter

Paul-Ward, Amy

Pauly, Vanessa

Pavagadhi, Shruti

Pavic, Aleksandar

Pavlopoulou, Ioanna

Pawlas, Natalia

Pazdro, Robert

Paz-Ferreiro, Jorge

Peace, Michelle

Pearce, Dora Claire

Pearson, James

Peck, Richard M.

Peckham, Erin

Pecurariu, Oana Falup

Peer, Nasheeta

Peerenboom, Peter Bob

Peijnenburg, Willie

Peller, Julie

Pelletier, Guillaume

Peltier, Richard

Peña Quintana, Luis

Peng, Szu-Hsien 
Peng, Chien-fang

Pengo, Martino

Penkala, Stefania

Pennock, Nathan

Pentakota, Sri Ram

Pereira, Dolores

Pereira-da-Silva, Luís

Pérez, Patricio

Pérez, Sandra

Pérez Ballesta, Pascual

Pérez Castrillón, José Luis

Pérez-Rodrigo, Carmen

Peri-Rotem, Nitzan

Perlmuter, Lawrence Charles

Perrella, Sharon L.

Perry, Lin

Perry, Yael

Persson Waye, Kerstin

Perumal, Deepak

Pes, Giovanni Mario

Petach, Helen

Peter, Richard

Petermans, Jean

Peters, Derek M.

Peters, Ruud J. B.

Peters, Ruth

Petersen, Kristina

Petersen, Eskild

Peterson, Donna Jean

Petrangeli Papini, Marco

Petrić, Dušan

Petrie, Joshua G.

Petroselli, Andrea

Petrusca, Daniela

Petry, Sebastian

Pettersson, Jean

Petti, Claudio

Pettinari, María Lucrecia

Petts, Richard J.

Pfeffer, Karen

Pfoertner, Timo-Kolja

Phan, Thai

Phibbs, Suzanne R.

Phillips, Carl

Phillips, Karen

Phillips, Judith

Phillips, Gregory

Phongsavan, Philayrath

Piao, Yunxian

Piatt, Gretchen A.

Piffaretti, Jean-Claude

Pignata, Cristina

Pignataro, Giuseppe

Pilcher, June

Pillai, Vijayan K.

Pineda, Paloma

Pinnapireddy, Shashank Reddy

Piotrowski, Caroline
Piperakis, Michael

Piqueras Rodríguez, José Antonio

Pircher Verdorfer, Armin

Pires, José Carlos Magalhães

Pirogova, Elena

Pirrone, Federica

Pistelli, Francesco

Pistone, Alessandro

Pithara, Christalla

Pitt, John

Pitteri, Marco

Pivonello, Rosario

Plain, Karren M.

Planck, Tereza

Plano, Daniel

Plumert, Jodie

Pochampally, Kishore K.

Podgorski, David C.

Poelman, Maartje P.

Poglayen, Giovanni

Pogson, Mark

Pokorn, Marko

Polanska, Kinga

Polen, Terry

Polesel, Fabio

Polidori, Guillaume

Polk, Deborah

Pollack, Keshia

Pollitt, Krystal

Polo-Kantola, Päivi

Polom, Karol

Polya, David

Pomatto, Mary Carol

Pompili, Maurizio

Pond, Dimity

Ponikau, Jens U.

Pontual, Emmanuel V.

Popp, Denny

Porsius, Jarry T.

Portegijs, Erja

Portengen, Lützen

Portnov, Boris

Postnova, Svetlana

Pottie, Kevin

Poulas, Konstantinos

Poulsen, Dorthe Varning

Pounder, Kieran

Pourchez, Jérémie

Pourret, Olivier

Powell-Wiley, Tiffany M.

Power, Thomas G.

Powers, James S.

Powles, John

Prado, Gustavo Faibischew

Prætorius, Thim

Pratt, Sarah

Pratt, Stephanie G.

Predieri, Guerrino 
Preece, Ellen P.

Preethichandra, Preethi

Prensner, John

Price-Wolf, Jennifer

Prigent, Amélie

Prince, Latrina

Prince, Bennie

Prior, Jerilynn C.

Procter, Sandra B.

Procter-Gray, Elizabeth

Proskocil, Becky J.

Prota, Francesco

Prunuske, Amy

Pryss, Rüdiger

Pueyo Anchuela, Óscar

Pujol, Sophie

Pulakka, Anna

Pulido, Olga M.

Pulignani, Silvia

Pulster, Erin

Punshon, Tracy

Purcell, Ninfa

Purmehdi, Mostafa

Puy, Jaume

Puzon, Geoffrey J.

Puzzolo, Elisa

Pyke, Chris

Pype, Marie-Laure

Qian, Wuyong

Qian, Qin

Qin, Bo

Qin, Quande

Qin, Kai

Qiu, Ruofeng

QIU, Jianying

$\mathrm{Qu}$, Shaojian

Quadros, Patricia Dörr De

Quarmby, Tom

Quevedo, Lluisa

Quinlan, Jennifer J.

Quinn, Ashlinn

Quisenberry, Amanda J.

Quock, Ryan

Rabinowitz, Samuel

Racine, Elizabeth

Rada, Elena Cristina

Radziemska, Maja

Rafaj, Peter

Ragas, Ad M. J.

Rage, Estelle

Rahman, Rahbel

Rahman, Aziz

Rajaee, Mozhgon

Rajeev, Sreekumari

Rajurkar, Mihir

Rakshit, Sudipta

Ralf, Risser

Ralston, Margaret
Ralston, Margaret L.

Ramakrishnan, Sreejith

Ramanathan, Subha

Ramirez, Marizen

Ramírez-Santana, Muriel

Ramos, Patricia

Ramos, Athena

Ramos Miras, Jose Joaquin

Ramsahoye, Bernard

Ramsay, Michael A.

Ramsden, Vivian

Ramström, Lars M.

Rana, Satiander

Rana, Dipak

Ranabhat, Chhabi

Ranadheera, Senaka

Rand, Matthew

Randall-Simpson, Janis

Ranjitkar, Sarbin

Ranmuthugala, Geetha

Rantakokko, Merja

Rantanen, Ville

Rao, Sheela

Rapp, Kilian

Rashedi, Ehsan

Rasul, Azad

Rato, Luís Pedro Ferreira

Ratschen, Elena

Raven, Melissa

Raynes-Greenow, Camille

Rayson, Gary D.

Redding, Colleen

Reddy, Ravi Krishnan

Reddy, Sandeep

Rediske, Richard R.

Reece, Lindsey

Reed, Derek

Reed, Edward Justy

Reeves, Aaron

Rege, Shraddha

Rehkopf, David

Rehman, Junaid U.

Rehman, Laurene

Reichmayr, Johannes

Reifsnider, Elizabeth

Reinhardt, Jan Dietrich

Reis, Stefan

Reisen, William K.

Reisi, Marzieh

Reiter, E. Miranda

Ren, Qingzhong

Ren, Yanfang

Ren, Jinma

Rengasamy, Samy

Renzetti, Claire M.

Requia, Weeberb J.

Réquia, Weeberb

Resch, Franz 
Reszka, Edyta

Reutfors, Johan

Rey, Jorge

Reyes, Jeanette

Reynolds, Amy

Reynolds, Lori

Rhoades, Martha

Ribeiro, Ana Isabel Correia

Ribeiro, Helena

Ribeiro, Ana Isabel

Ribeiro, Edna

Ricceri, Fulvio

Riccio, Paolo

Riccio, Angelo

Riccio, Giuseppe

Rice, Penelope A.

Rice, Robert H.

Rice, Mark

Richards, Laura

Richardson, Clive

Richmond, Janet

Richter, Kneginja

Riedel, Natalie

Riediger, Natalie D.

Rietra, Rene

Rigby, Patricia

Riggs, Elisha

Rigotti, Thomas

Riiser, Amund

Rikard, R. V.

Riley, Karin L.

Riley, Lee W.

Rimondini, Lia

Riquelme, Adrián

Riveron, Jacob

Robbins, Miriam R.

Roberts, Jennifer D.

Robichaud, Alain

Robins, Alan

Robinson, Lisa

Robles, Luke

Rocklöv, Joacim

Rocklöv, Åsa Holmner

Röder, Stefan

Roderick, Paul

Rodgers, Rachel F.

Rodrigo-Comino, Jesús

Rodrigues, Jean-Marie

Rodríguez, Jesús Álvarez

Rodriguez-Rodriguez, Fernando

Rodríguez-Seijo, Andres

Rodziewicz, Joanna

Roebuck, Bill

Roest, Kees

Roex, Erwin

Rogers, Todd

Rogerson, Mike

Rohde, Nicholas
Rojas-Rueda, David

Romani, Massimo

Romano, Megan

Romano, Nicla

Romano, Audrey

Romano, Megan

Romanowski, Kathleen S.

Romer, Dan

Romero, Inmaculada

Room, Robin

Root, Martin M.

Rosa, Maria José

Rosário, Rafaela

Rosenbluth, Jennifer M.

Ross, Veerle

Rossi, Paolo Giorgi

Rossi, René M.

Rostaing, Lionel

Roswall, Nina

Rott, Eduard

Roumpakis, Antonios

Rovira, Pere

Rowlinson, Steve

Roy, Denis

Różalska, Barbara

Rozsival, Pavel

Ruano Ravina, Alberto

Rubinelli, Sara

Rucci, Paola

Rüegg, Joelle

Ruffalo, Leslie

Ruiz-Canela, Miguel

Ruiz-García, Alejandro

Ruiz-Narváez, Edward A.

Ruiz-Robledillo, Nicolás

Rummo, Pasquale

Ruscica, Massimiliano

Russell, Joanna

Russell, Marie

Russell, Armistead

Russell, Fraser D.

Rust, Michael

Rüttiger, Lukas

Ryabov, Igor

Ryan, Michael

Ryder, Sheila

Rydstedt, Leif

Ryser, Elliot

Saad, Mohamad

Sacande, Moctar

Sachmechi, Issac

Sacristán, Daniel

Sádaba, Charo

Sadat, Jasmin

Saddik, Basema

Sadek, Hesham A.

Sadler, Euan

Saez, Marc 
Safavi, Kamran

Sagebiel, John C.

Saha, Sanjib

Saha, Shubhayu

Sainani, Kristin

Sainz, Carlos

Saito, Kan

Sajobi, Tolulope

Sakai, Toshio

Sakakibara, Masayuki

Sakan, Sanja M.

Sakata, Masahiro

Sakellariou, Dikaios

Sakhaee, Khashayar

Saksena, Sumeet

Sakumura, Yuichi

Sala, Roser

Salahudeen, Mohammed

Salaris, Luisa

Salas, Lucas A.

Salazar, Alejandro

Salazar, Natalia Villota

Salazar, Leslie Ramos

Salazar, Debra J.

Salgado, Cassandra D.

Sallam, Mohamed F.

Salm, Adrienne K.

Salmon, Charles Thomas

Salom, Scott

Salter, Linda-Ruth

Samadi, Sayyed Ali

Sambamoorthi, Usha

Sambri, Vittorio

Sammartino, Maria Pia

Sampson, Natalie R.

San Jose, Roberto

Sandbæk, Mona

Sandra, Jaksic

Sandy, Larissa

Sankaranarayanan, Nehru Viji

Sankavaram, Kavitha

Sanmiquel Pera, Lluis

Sano, Daisuke

Santamaria, Rita

Santarelli, Lory

Santikarn, Chamaiparn

Santilio, Angela

Santos, Maria Teresa L. Dos

Santos, Maria Jacinta

Santos, Maria Paula

Santos Zanchetta, Margareth

Santos-Gallego, Carlos

Sardaro, Ruggiero

Sargentis, George-Fivos

Sarkar, Sumit

Sarpong, Daniel F.

Sasseville, Denis

Satchidanand, Nikhil
Saunders, Daniel G.

Saurina, Carme

Savelyev, Alexander

Savoie Roskos, Mateja

Sawa, Teiji

Sbihi, Hind

Scannell Bryan, Molly

Scasny, Milan

Schaffner, Donald

Schalinski, Inga

Schechter, Julia Corwin

Scheepers, Paul T. J.

Schembari, Anna

Schepelmann, Philipp

Scherr, Sebastian

Schieweck, Alexandra

Schiff, Miriam

Schilaty, Nathan

Schimmenti, Adriano

Schindler, Stefan

Schiza, Sophia E.

Schmalwieser, Alois W.

Schmeling, Andreas

Schmeltz, Michael T.

Schmidt, Michael

Schmidt, Amand

Schmidt, Christina

Schmidt, Silke

Schmitt, Laetitia Helene Marie

Schneeweis, Adina

Schneider, Petra

Schneider, Alexandra

Schnell, Izhak

Schoenhagen, Paul

Schomer, Paul

Schonthal, Axel

Schöpfer, Jutta

Schreiner, Pamela

Schroeckh, Volker

Schroeder, Megan J

Schroeder, Winfried

Schubert, Sebastian

Schubert, Jochen

Schullehner, Jörg

Schultz, Andre

Schulz, Peter J.

Schulze, Matthias B.

Schumann, Barbara

Schutte, Anne

Schwab, Keri

Schwartz, Gary

Schwartz, Brian

Schwarz, Eli

Schweitzer, Dietrich

Scortichini, Matteo

Scott, Pippa

Scott, Britain

Scott, Stephanie 
Scott, Iain

Scotti, Marcus

Scrine, Clair

Seaman, Kate

Sears, Louis

Seckmeyer, Gunther

Secondi, Luca

Seebauer, Sebastian

Seeman, Mary V.

Segura-Ortí, Eva

Seidel, Michael

Seixas, Adérito

Sekine, Yoshika

Sell, Katie

Selland, Corey

Sellarés, Jacob

Sellix, Michael T.

Selvamani, Amutha

Selya, Arielle

Sembajwe, Grace

Semmens, Erin

Sera, Francesco

Serafini, Gianluca

Serpico, Rosario

Serrão, José Eduardo

Servadio, Pieranna

Servidio, Rocco

Seth, Devanshi

Setti, Ilaria

Setyan, Ari

Severine, Deguen

Sha, Zhe

Shaban, Ramon Z.

Shah, Tayyab

Shahabfar, Alireza

Shahid, Saroash

Shahid, Rizwan

Shahmoradi, Mahdi

Shaker, Richard

Shamasunder, Bhavna

Shang, Ce

Shankar, Aparna

Shanmugam, Muruganandan

Shao, Zhenfeng

Sharan, Riti

Shareck, Martine

Sharma, Rohit

Shaw, Rajib

Shaw, Richard J.

Sheehan, Dianna

Sheikh, Sophia S.

Shelton, Nicola

Shen, Jiabin

Shen, Jun

Shen, Zhenjiang

Shen, Suwan

Sheppard, Paula

Sheppard, Amanda J.
Sherchan, Samendra

Sherratt, Fred

Shewale, Swapnil

Shi, Baoyou

Shi, Victor

Shi, Yuan

Shi, Yuyan

Shi, Huijing

Shiau, Tzay-An

Shih, Patrick C.

Shil, Niraj

Shim, Jaejun

Shim, Sang-Oh

Shimek, Jo Anna

Shin, Won Sop

Shin, Dong-chun

Shin, Dayeon

Shishegar, Nastaran

Shoffstall, Andrew J.

Shoji, Tsuchida

Shoji, Kotaro

Shoji, Kensuke

Shook, Allan

Shyr, Oliver F.

$\mathrm{Si}$, Lei

Sicard, Pierre

Siceloff, E. Rebekah

Siceloff, Rebekah

Sicotte, Diane

Siddall, Andy

Sieber, Nancy

Siega-Riz, Anna Maria

Siegel, Robert

Sienkiewicz, Zenon

Sigler, Jeffrey

Signorelli, Salvatore Santo

Sigurvinsdottir, Rannveig S.

Silfee, Valerie

Sillanpää, Mika

Silva, Gabriela Jorge

Silva, Lígia

Silva, Cristiano M.

Silvan, Jose Manuel

Sim, Kyra

Sim, Chang Sun

Simmering, Jacob E.

Simões, Marta Filipa

Simon, Marie-Christine

Simonds, Vanessa

Simons, Shellie R.

Simplican, Stacy Clifford

Sims, Jennifer

Sims, Jane

Sinclair, Ryan

Sinden, Kathryn

Sindi, Shireen

Singer, Andrew C.

Singh, Atul Kumar 
Singh, Harsimran

Singh, Sanjay

Singh, Neenu

Singleton, Chelsea

Singovszka, Eva

Siniscalco, Dario

Sioris, Christopher

Siraj, Amir S.

Sirca, Costantino

Sirić, Ivan

Sirsat, Sujata

Sit, Cindy

Sivaram, Sudha

Sjödin, Fredrik

Skärbäck, Erik

Skelin, Ana Kuzmanić

Skoczko, Iwona

Skouloudis, Andreas N.

Skrzypiec, Grace

Skwarzec, Bogdan

Slack, Frances

Slaughter, James

Sloan, Chantel

Sloan, Robert Alan

Slot, Jason C.

Smarr, Melissa

Smeets, Patrick W. M. H.

Smit, Brandon W.

Smith, Thomas

Smith, Stuart

Smith, Leonard B.

Smith, Andy

Smith, Brittany L.

Smith, David I.

Smith, Ryan C.

Smith, Julie A.

Smith, Genee S.

Smith, Miichael (Mike)

Smythe, David

Snijders, Deborah

Snitker, Soren

Snyder, Eric

Soares, Paula

Sobota, Aleksander

Socol, Yehoshua

Sohaib, Osama

Soiza, Roy L.

Sojic, Aleksandra

Sokal-Gutierrez, Karen

Soles, Gillian

Solo-Gabriele, Helena

Solomon, Patty

Solovyev, Nikolay

Sommer, Harald

Sone, Hirohito

Sone, Hideko

Song, Fujian

Song, Guo
Song, Ranran

Song, Jinxi

Soos, Istvan

Sornay-Rendu, Elisabeth

Soudeyns, Hugo

Soukupová, Jana

Soulage, Christophe

Soultos, Nikolaos

Soundy, Andy

Sousa, Carla A.

Sousa, Joao Carlos

Sousa, Nelson

Sousa Fragoso, Rui

Southworth, Michael

Sowa-Kofta, Agnieszka

Spangler, John

Spear, Suzanne E.

Spencer-Hwang, Rhonda

Sperlich, Birgit

Spieckermann, Sven

Spilanis, Ioannis

Spinazzè, Andrea

Spires, Steven

Spitz, Lewis

Spörndly, Rolf

Springgate, Ben

Spüler, Martin

St Clair, Michelle C.

St John, James A.

St. Hilaire, Melissa A.

St.Helen, Gideon

Stabile, Luca

Stacey, Brandon

Stack, Steven

Stadtlander, Timo

Stafoggia, Massimo

Stagnitti, Karen

Stagos, Dimitrios

Stahl Jr., Ralph G.

Staley, Zachery R.

Stallings-Smith, Sericea

Stalsberg, Helge

Stam, Rianne

Stamatis, Nikolaos

Stamey, James D.

Stanca, Sarmiza Elena

Stanek, Agata

Stanišić Stojić, Svetlana

Stanistreet, Debbi

Stanistreet, Debbi

Stark, Emily

Starr, Martha A.

Stathis, Dimitrios

Stauvermann, Peter Josef

Stavi, Ilan

Stavropoulos-Kalinoglou, Antonios

Stearns, Jodie A.

Stearns, Diane 
Stedman-Smith, Maggie

Steenkamp, Malinda

Steeves, Betsy Anderson

Stefanakis, Alexandros

Stehlik, Daniela

Stehr, Mark

Stein, Robert M.

Stelmach-Mardas, Marta

Stephens, Alexandre

Stermac, Lana

Stevens, David

Stevinson, Clare

Stewart, Anna M.

Stewart, Alan E.

Stewart, Barclay T.

Sticca, Fabio

Stiehl, Emily

Stilianakis, Nikolaos I.

Stillman, Martin

Stingone, Jeanette

Stins, John F.

Stivrins, Normunds

Stockings, Emily

Stoehr, Tobias

Stoforos, Nikolaos G.

Stoltz, Jonathan

Stolz, Erwin

Stone, Andrea

Stoneham, Melissa

Stoner, Lee

Stortini, Angela Maria

Stout, David A.

Strady, Emilie

Strong, Carol

Strubelt, Henning

Stylos, Nikolaos

Su, Hsin-Ting

$\mathrm{Su}$, Shiliang

Su, Shaoyong

$\mathrm{Su}$, Yuan-Fong

Subbiah, Murugan

Subhan, Fatheema Begum

Subramaniam, Prasad

Subramanian, Devika

Sudano, Laura Elizabeth

Sueki, Hajime

Suen, Sze-chuan

Sugg, Maggie M.

Suggs, Suzanne

Sugiyam, Koji

Sugiyama, Takemi

Suh, Dong Hee

Suhaili Ramli, Nur

Sukumaran, Anil

Sullivan, Elinor L.

Sullivan, Marianne

Sumner, Walton

Sun, Quanfu
Sun, Sun

Sun, Ling

Sun, Dianjianyi

Sun, Li-Yun

Sun, Weiling

Sun, Zhifu

Sun, Wenjie

Sundaram, Rajeshwari

Suñer Soler, Maria Rosa

Sung, Jidong

Suo, Weilan

Sureda, Francisca

Sussan, Thomas

Sutaria, Dhruvitkumar

Sutphin, Suzanne

Sutton, Jeannette

Suurmond, Jeanine

Suwazono, Yasushi

Svanemyr, Joar

Svartengren, Magnus

Svensson, Jannet

Svob, Connie

Swann, Brian Jeffrey

Swanson, John

Swenson, Cynthia Cupit

Świątkowska, Beata

Sy, Ibrahima

Sygit, Katarzyna

Symonds, Erin M.

Symons, Martyn

Szafron, Michael

Szekely, Gyorgy

Székely, Andrea

Szulczewska, Barbara

Szymańska-Pulikowska, Agata

Szyszkowicz, Mieczysław

Tadić, Vanja

Takada, Satoru

Takahashi, Ken

Takahashi, Hajime

Takahisa, Kanekiyo

Takala, Jukka

Takamura, Noboru

Takano, Tomomi

Takebayashi, Hideki

Takebayashi, Shigeo

Taliaferro, Lindsay

Tall, Ben D.

Tammelin, Tuija

Tamosaitiene, Jolanta

Tamulevicius, Nauris

Tamura, Atsutaka

Tan, Chun Liang

Tanabe, Masaki

Tang, Xiaochen

Tang, Jianjun

Tang, Robert

Tang, Yilang 
Tang, Naijun

Tang, Meng-Chi

Tanigawa, Koichi

Tanner, Grit

Tanton, Robert

Tao, Shasha

Tao, Yan

Taplin, Stephanie

Tarabieh, Khaled

Tarazona-Santabalbina, Francisco José Osé

Taren, Douglas

Targiel, Krzysztof

Tarlov, Elizabeth

Tarnoki, Adam Domonkos

Tartakovsky, Leonid

Tasmin, Saira

Tavernier, Jan

Tavori, Hagai

Tawia, Susan

Taylor, Graham

Taylor, Cristopher M.

Taylor-Robinson, Andrew W.

Tchangani, Ayeley P.

Te, Shu Harn

Tecles, Fernando

Tefft, Nathan

Teismann, Tobias

Teixeira, Samantha

Telfair, Joseph

Teli, Despoina

Tempera, Italo

Temple, Norman

Templeton, Michael

Tenkate, Thomas

Teras, Lauren R.

Terblanche, Lourie

Terry, Daniel

Tervonen, Hanna

Teske, Jennifer

Tesler, Riki

Testoni, Ines

Tetrick, Lois E

Thai, Phong

Thanavala, Yasmin

Thapa, Subash

Theodorsson, Gudjon Elvar

Theorell, Töres

Theorell-Haglöw, Jenny

Thiara, Ravi

Thibodeau, Alexandre

Thibodeaux, Terry

Thiel, Cassandra L.

Thielemann, Christiane

Thielens, Arno

Thigpen, Calvin

Thikkurissy, Sarat

Thøgersen-Ntoumani, Cecilie

Thoma, Myriam
Thomas, Roger E.

Thomas, Kevin

Thomas, Stephen B.

Thomas, Elizabeth A.

Thompson, Wendy

Thompson, Andrew

Thorsteinsson, Einar

Thrane, Ulf

Thunders, Michelle

Thurber, Mark

Thuy, La Thi Thanh

Tian, Yilin

Tiefelsdorf, Michael

Tiehm, Andreas

Tigbe, William

Timmermans, Luc

Timmermans, Harry

Timmins, Kate

Tin Tin, Sandar

Tinnerberg, Håkan

Tippett, Vivienne

Toccolini, Alessandro

Tochkov, Kiril

Toderi, Stefano

Togo, Fumiharu

Toivanen, Susanna

Tokoro, Chiharu

Tomaselli, Venera

Tomášková, Hana

Tomaszewska, Ewa

Tomata, Yasutake

Tomczak, Andrzej

Tomioka, Michiyo

Tommasino, Luigi

Ton, Shan-Shin

Tong, Liang

Tong, Zheming

Tong, Zhijun

Tong, Hoang V.

Toniolo, Antonio Q.

Toomey, Clodagh

Torio, Celeste Marie

Toropova, Alla

Torres, Elisa

Torres, João Paulo

Torres De Oliviera, Rui

Torres Lagares, Daniel

Torres López, Ma Isabel

Torres-Barceló, Clara

Torres-Padrón, M. Esther

Toscano, William

Toumba, Meropi

Tountas, Yannis

Towne, Samuel D.

Townsend, Kristy

Tran, Huy

Trawley, Steven

Trevisan, Andrea 
Trinh, Linda

Trovato, Francesca Maria

Trudeau, Francois

True, Larissa

Trulson, Chad R.

Tsagarakis, Konstantinos

Tsai, Chen-Chi

Tsai, Jing-Jane

Tsai, Li-Tang

Tsao, Lee-Ing

Tsapakis, Ioannis

Tseles, Dimitrios

Tseng, Yi-Ping

Tseng, Tung-Sung

Tseng, Chun-Mao

Tseng, Hsu-Min

Tsiamis, Costas

Tsikouras, Panagiotis

Tsionas, Mike G.

Tsitsos, William

Tso, For Yue

Tsourtos, George

Tsow, Francis (Tsing)

Tsugane, Shoichiro

Tucker, John

Tume, Pedro

Tuomisto, Jouko

Turenne, Christine Y.

Turgeon, Nathalie

Turner, Michelle

Tutka, Piotr

Tykkyläinen, Markku

Tytherleigh, Michelle

Tytła, Malwina

Tzanis, Chris

Tziomalos, Konstantinos

Uhl, Eberhard

Ulibarri, Gerard

Upshur, Carole C.

Urban, Aleš

Urrestarazu, Elena

Urschler, David

Urzi Brancati, Cesira

Usai, Alessia

Uusi-Rasi, Kirsti

Uzu, Gaëlle

Vaccarini, Massimo

Vaccarini, Katiuscia

Vacchina, Véronique

Vafeiadi, Marina

Vaiman, Daniel

Vakulin, Andrew

Valanne, Susanna

Valentukeviciene, Marina

Valeo, Caterina

Valério, Elisabete

Valério Monteiro, Magda Sofia

Vallejo Ruiz, Asier
Vallone, Donna M.

Vallone, Donna

Valtorta, Nicole

Van, Thinh Nguyen

Van Den Berg, Maayken

Van Den Ende, Jef

Van Der Ende, P(eter). C. Van Der Wel, Kjetil A.

van Dijk, Jitse

Van Dijk, Meine Pieter

Van Doorn, Robert

Van Hees, Vincent

Van Hoof, Joost

Van Hoof, Joris J.

Van Orden, Kimberly

Van Rossem, Lenie

Van Stempvoort, Dale R.

Vance, Marina

Vance, Eric

Vandenberg, Ann E.

Vanderslice, Robert

VanDerslice, Jim

Vanscheeuwijck, Patrick

Vardasca, Ricardo

Varnes, Julia R.

Varrica, Daniela

Vasel, Jean-luc

Vasileiou, Konstantina

Vaughn, Allison

Vaz Moreira, Ivone

Vaz-Moreira, Ivone

Vázquez Duhalt, Rafael

Vedøy, Tord Finne

Veeranki, S. Phani

Vega, Claudia

Velasco Garrido, Marcial

Velasco Ortega, Eugenio

Venables, Dan

Venette, Steven J.

Venn, Bernard

Venter, Carina

Venturelli, Massimo

Venturini, Elisa

Verani, Marco

Verd, Sergio

Verdam, Mathilde

Verdeguer, Francisco

Vergara, Lizandra Garcia Lupi

Verhoeven, Adrie

Verhoughstraete, Marc

Vermeir, Gerrit

Verriele, Marie

Verrotti, Alberto

Vesper, Stephen

Vetter, Sylvia Helga

Viazzi, Francesca

Vidal, Tânia

Viegas, Carla 
Vieira, João

Vijayan, Murali

Vik, Tennley

Vilaro, Melissa J.

Villalobos, Bianca

Villalonga-Olives, Ester

Vimercati, Luigi

Vincent, Grace

Violanti, John M.

Virtanen, Jorma I.

Visentin, Denis

Vita, Roberto

Vitali, Matteo

Vitas, Ana Isabel

Vitztum, Coley

Vivat, Bella

Vivili, Paula

Vo, Minh D.

Voaklander, Donald C.

Vogt, Bruno

Volavka, Jan

Volken, Thomas

Volland, Gerhard

Volpe, Richard

Von Lewinski, Dirk

Vosvick, Mark A.

Voudris, Vassilis

Voulvoulis, Nick

Vozza, Iole

Vuckovic, Milena

Vuillermoz, Cecile

Vuorio, Alpo

Vuyst, Stijn

Wade, Charles E.

Wagenfeld, Amy E.

Waibel, Christian

Waite, Sue

Walabyeki, Julie

Walcek, Chris

Wałdykowski, Piotr

Walker, D. Catherine

Walker, Ella

Walker, Ana Paula

Wallace, Maeve

Waller, Lance

Walser, Sandra M.

Walsh, Shana

Walters, Vicky

Walton, AnnMarie L.

Walton, AnnMarie

Walz, Yvonne

Wan, Shuping

Wan, Zhanming

Wan Mohd Yunus, Wan Mohd Azam

Wanchai, Ausanee

Wang, Lian-Sheng

Wang, Daowen

Wang, Yang
Wang, Zheng-Xin

Wang, Yuling

Wang, Ming-Hua

Wang, Jianying

Wang, Chenghao

Wang, Di

Wang, Jianxu

Wang, Ruoniu

Wang, Dong

Wang, Jinfeng

Wang, Zhengxin

Wang, Shigong

Wang, Grace Y

Wang, Junbo

Wang, Mo

Wang, Feng

Wang, Yinhe

Wang, Aolin

Wang, Haitian

Wang, Hsiao-hsuan

Wang, Ping

Wang, Wei

Wang, Shengrui

Wang, Li-Yu

Wang, Xiang

Wang, Jiasheng

Wang, Zhensheng

Wang, Qun

Wang, Jiejing

Wang, Litao

Waples, James

Warber, Sara

Ward, Kenneth D.

Ward, Peter M.

Ward, Emma

Wark, Stuart

Warmoth, Krystal

Watanabe, Toru

Watanabe, Tsunemi

Waters, Catherine M.

Waters, Jane

Waters, Matthew

Watkins, Kari

Wątróbski, Jarosław

Watt, Peter

Weaver, Scott

Weaver, Louise

Weaver, Matthew

Webb, Tom

Webb, Cameron E.

Webb, Elizabeth

Weber, Ellerie S.

Webster, Noah J.

Wecht, Jill

Wege, Natalia

Wei, Chao-Yang

Wei, Benyong

Wei, Da-Hua 
Wei, Ye

Wei, Binggan

Weidhaas, Jennifer

Weinberg, Seth

Weiner, Judith

Weiner, Shira Schecter

Weis, Megan A.

Weismayer, Christian

Weiss, Sabrina

Weiss, William

Weitkamp, Gerd

Weker, Halina

Welch, David

Welden, Natalie

Welgamage Don, Aakash

Welles, Seth

Wells, Ellen

Wells, Brian J.

Wells, Nancy

Wells, Deborah

Welzel, Tyson

Weng, Chingfeng

West, Suzanne L.

Westerlund, Anna

Westphal, Andreas

Westrupp, Elizabeth Mary

White, Kim

White, Nicole

White, Scott M.

White, Wendy

Whitehouse, Hilary

Whiteley, Hugh

Whittaker, Peter

Whittemore, Katherine

Wickliffe, Jeffrey

Widenmeyer, Marc

Wieczorowska-Tobis, Katarzyna

Wiedemann, Florian

Wielgomas, Bartosz

Wiernik, Brenton M.

Wierzejska, Regina

Wieser, Simon

Wiesmeier, Martin

Wiggins, Brenda P.

Wiggs, Michael

Wiklund Axelsson, Sarianne

Wilburn, Kathleen M.

Wilde, Annegret

Wildman, John

Wilking, Cara

Williams, Joah L.

Williams, Adina

Williams, Matt

Williamson, Grant

Wilson, Anne M.

Wilson, Debra Rose

Wilson, Philip

Wilson, Fernando A.
Wilson, Peter

Wilson, Mark J.

Windham, Gayle

Winford, Eboni

Winkler, Daniel E.

Winters, Shannon

Winters, Stephen

Winwood, Peter

Wirth, Michael

Witorsch, Raphael Jay

Wittlich, Marc

Wohlfarth, Rainer

Wójkowska-Mach, Jadwiga

Wollesen, Bettina

Won, Jae Woong

Wong, Vincent Kam Wai

Wong, Jen D.

Wong, Grace Lai-Hung

Wong, Ling Tim

Wong, Cynthia

Woo, Jong Min

Wood, Jen

Woodfield, Lorayne

Woodrow, Chris

Woods-Chabane, Gwen C.

Woods-Townsend, Kathryn

Woodward, Alistair Jack

Woolcock, Geoff

Wotschack, Philip

Wovkulich, Karen

Wright, Kamau

Wright-Berryman, Jennifer

$\mathrm{Wu}$, Chih-Da

$\mathrm{Wu}$, Yanyan

Wu, Liyun

$\mathrm{Wu}$, Hung-Yi

$\mathrm{Wu}$, Jianbo

$\mathrm{Wu}$, Yonghua

$\mathrm{Wu}$, Kevin Chien-Chang

$\mathrm{Wu}$, Weining

$\mathrm{Wu}$, Lifeng

$\mathrm{Wu}, \mathrm{Qi}$

$\mathrm{Wu}$, Alan $\mathrm{Hb}$

Wuppermann, Amelie C.

Wynne, Olivia

Wyver, Shirley

Xi, Junqiang

Xia, Ting

Xia, Yankai

Xia, Meimei

Xia, Ningshao

Xia, Yuchen

Xiang, Yisha

Xiang, Jianjun

Xiang, Yu

Xiaohui, $\mathrm{Xu}$

Xiaoli, Alus

Xie, Xiaoyao 
Xie, Grace

Xie, Jin Gui

Xie, Shaohua

Xing, Yangang

Xiong, Zhaohui

$\mathrm{Xu}, \mathrm{Xun}$

$\mathrm{Xu}$, Yongyong

$\mathrm{Xu}$, Xiaohui

$\mathrm{Xu}, \mathrm{Xin}$

$\mathrm{Xu}$, Iris

$\mathrm{Xu}$, Zhiwei

Xu, Yuan

Xuan, Tran Dang

Xue, Jinkai

YAGER, ERIC

Yamada, Tetsuji

Yamamoto, Naomichi

Yang, Xiaomei

Yang, Zhongping

Yang, Linsheng

Yang, Feng

Yang, Kwang Ik

Yang, Xia

Yang, Yong

Yang, Xin

Yang, Min

Yang, Fan

Yang, Haining

Yang, Jiachuan

Yannopoulos, Stavros

Yano, Takashi

Yao, YuChun

Yassi, Annalee

Yates, Nathanael J.

Ye, Ming

Yearwood, Edilma L.

Yeckel, Catherine Weikart

Yee, Susan

Yeh, Chih-ko

Yeh, Chia-Tsung

Yeh, Tsu-Ming

Yeh, Shu-Hui

Yen, Yee-wen

Yeo, Younsook Anna

Yershova, Kseniya

Yi, Jean C.

Yiannakoulias, Niko

Yiin, Lih-Ming

Yilmaz, Kuzey

Yin, Jingjing

Ying, Zhekang

Yokoyama, Yoko

Yoo, Do Guen

Yoo, Tag Keun

Yoo, Hong Il

Yoon, Sukhwan

Yoon, Jiyoon

Yoshiki, Syuji
Yoshinaga, Jun

Yoshinaga, Jun

Yoshizumi, Terry

You, Wen

You, Kefei

You, Xiaona

Young, Sean G.

Young, Jeanine

Young, Sean

Yousefzadeh, Sepideh

Youssef, Noha

Yperman, Jan

Yu, Xiaosong

Yu, Jin-Gang

$\mathrm{Yu}$, Tsung-Hsien

Yu, Chia-Yuan

Yu, Rongjie

Yu, Jae-Hyuk

Yu, Ruby

Yu, Chia-Pin

Yuan, Quan

Yuan, Chao-Qing

Yuan, Pao-Chiang

Yuan, Xue

Yuan, Haoran

Yue, Qi

Yue, Xiaohang

Yuen, Belinda

Yuen, Hon K.

Yurasek, Ali M.

Yurekli, Ayda

Zabka, Martin

Zaborskis, Apolinaras

Zabucchi, Giuliano

Zaccheo, Patrizia

Zaharia, Carmen

Zalouk-Vergnoux, Aurore

Zambrano, Luis

Zanini, Barbara

Zarfel, Gernot E.

Zavadskas, Edmundas Kazimieras

Zeiner, Michaela

Zeman, Daniel Meier

Zempila, Melina Maria

Zeng, Guangming

Zeng, Huiping

Zeng, Di

Zeng, Shihong

Zeni, Olga

Zhang, Jian

Zhang, Wei

Zhang, Jay

Zhang, Zengqiang

Zhang, Tao

Zhang, Jingwen

Zhang, Chen

Zhang, Bing

Zhang, Yusheng 
Zhang, Quin

Zhang, Yang

Zhang, Panyue

Zhang, Zhiyong

Zhang, Junzeng

Zhang, Dongfeng

Zhang, Ying

Zhang, Deqiang

Zhang, Zhuo

Zhang, Haifeng

Zhang, Rongjun

Zhang, Aiyong

Zhang, Zhenhua

Zhao, Xianhui

Zhao, Yun

Zhao, Yaqian

Zhao, Weifeng

Zhao, Qiran

Zhao, Yichuan

Zhao, Wenbing

Zheng, Xiaoying

Zheng, Tongzhang

Zhong, Weihong

Zhou, Lifeng

Zhou, Qingxiang

Zhou, Luxi

Zhou, An-Nan

Zhou, Chengchao
Zhou, Ligang

Zhu, Yuming

Zhu, Zhenduo

Zhu, Hong

Zhu, Xiaoshan

Zhu, Lin

Zhu, Shijun

Zhuang, Jie

Zhuang, Ziqing

Zhurakhovska, Lilia

Ziegler, Jane

Zielenkiewicz, Urszula

Zielinski-Gutierrez, Emily

Zijlema, Wilma

Zilberman, Uri

Zimeri, Anne Marie

Zimmerman, Emily

Zineldin, Mosad

Zou, Enmin

Zouharova, Monika

Źróbek-Sokolnik, Anna

Żubrowska-Sudoł, Monika

Zucca, Paolo

Züllig, Richard

Zuniga-Teran, Adriana

ZUSMAN, Eric

Zweifel, Peter

Zwoździak, Anna

(C) 2018 by the authors. Licensee MDPI, Basel, Switzerland. This article is an open access article distributed under the terms and conditions of the Creative Commons Attribution (CC BY) license (http://creativecommons.org/licenses/by/4.0/). 